\title{
Temporal changes of the flare activity of Proxima Centauri ${ }^{\star}$
}

\author{
Ya. V. Pavlenko ${ }^{1,2,3}$, A. Suárez Mascareño ${ }^{4}$, M. R. Zapatero Osorio ${ }^{5}$, R. Rebolo ${ }^{2,6,7}$, \\ N. Lodieu ${ }^{2,6}$, V. J. S. Béjar ${ }^{2,6}$, J. I. González Hernández ${ }^{2,6}$, and M. Mohorian ${ }^{8}$ \\ ${ }^{1}$ Main Astronomical Observatory of the National Academy of Sciences of Ukraine, 27 Zanolotnoho, Kyiv 03143, Ukraine \\ e-mail: yp@mao.kiev.ua \\ ${ }^{2}$ Instituto de Astrofísica de Canarias (IAC), Calle Vía Láctea s/n, 38200 La Laguna, Tenerife, Spain \\ 3 Center for Astrophysics Research, University of Hertfordshire, College Lane, Hatfield, Hertfordshire AL10 9AB, UK \\ ${ }^{4}$ Observatoire Astronomique de l'Université de Genève, 1290 Versoix, Genève, Switzerland \\ ${ }^{5}$ Centro de Astrobiología (CSIC-INTA), Carretera de Ajalvir km 4, 28850 Torrejón de Ardoz, Madrid, Spain \\ ${ }^{6}$ Departamento de Astrofísica, Universidad de La Laguna (ULL), 38205 La Laguna, Tenerife, Spain \\ ${ }^{7}$ Consejo Superior de Investigaciones Científicas, CSIC, Spain \\ ${ }^{8}$ Taras Shevchenko National University of Kyiv, Kyiv, Ukraine
}

Received 17 September 2018 / Accepted 9 May 2019

\begin{abstract}
Context. We study temporal variations of the emission lines of $\mathrm{H}_{\alpha}, \mathrm{H}_{\epsilon}, \mathrm{H}$ and $\mathrm{K} \mathrm{CaII}, \mathrm{D}_{1}$ and $\mathrm{D}_{2} \mathrm{NaI}_{1} \mathrm{He}_{4026}$, and $\mathrm{He}_{5876}$ in the HARPS spectra of Proxima Centauri across an extended time of 13.2 yr, from May 27, 2004 to September 30, 2017.

Aims. We analyse the common behaviour and differences in the intensities and profiles of different emission lines in flare and quiet modes of Proxima activity.

Methods. We compare the pseudo-equivalent widths (pEW) and profiles of the emission lines in the HARPS high-resolution $(R \sim 115000)$ spectra observed at the same epochs.

Results. All emission lines show variability with a timescale of at least $10 \mathrm{~min}$. The strength of all lines except $\mathrm{He}_{4026}$ correlate with $\mathrm{H}_{\alpha}$. During strong flares the "red asymmetry" appears in the $\mathrm{H}_{\alpha}$ emission line indicating the infall of hot condensed matter into the chromosphere with velocities greater than $100 \mathrm{~km} \mathrm{~s}^{-1}$ disturbing chromospheric layers. As a result, the strength of the CaII lines anti-correlates with $\mathrm{H}_{\alpha}$ during strong flares. The HeI lines at 4026 and $5876 \AA$ appear in the strong flares. The cores of $\mathrm{D}_{1}$ and $\mathrm{D}_{2} \mathrm{NaI}$ lines are also seen in emission. During the minimum activity of Proxima Centauri, CaII lines and $\mathrm{H}_{\epsilon}$ almost disappear while the blue part of the NaI emission lines is affected by the absorption in the extending and condensing flows.

Conclusions. We see different behaviour of emission lines formed in the flare regions and chromosphere. Chromosphere layers of Proxima Cen are likely heated by the flare events; these layers are cooled in the "non-flare" mode. The self-absorption structures in cores of our emission lines vary with time due to the presence of a complicated system of inward and outward matter flows in the absorbing layers.
\end{abstract}

Key words. stars: activity - stars: atmospheres - stars: chromospheres - stars: flare - stars: individual: Proxima Cen

\section{Introduction}

In this paper we define "flare activity" as a strong and timevariable phenomenon observed in stellar spectra and caused by the dissipation of magnetic field originated by dynamo processes in the stellar photosphere, in the chromosphere, or in the corona (e.g. Parker 1955). These processes differ comparatively from the case of slow phenomena of "stellar activity" responsible for variations of observed fluxes due to comparatively slow variations of spots and filament structures on the stellar surface. Observationally, the dynamo mechanism can be probed through the relationship between rotation and magnetic activity, and the evolution of these properties (Suárez Mascareño et al. 2015; Newton et al. 2017).

Magnetic activity in late-type stars is often measured in terms of $\mathrm{H}_{\alpha}$ emission and increases with decreasing stellar mass (e.g. Hawley et al. 1996). Magnetic activity increases with decreasing stellar mass; for example, Hawley et al. (1996). In addition, magnetically active stars are known to flare more

\footnotetext{
* Table A.1 is only available at the CDS via anonymous ftp to cdsarc.u-strasbg.fr (130.79.128.5) or via http://cdsarc. u-strasbg.fr/viz-bin/qcat?]/A+A/626/A111
}

frequently than inactive stars. Magnetic activity, stellar rotation and magnetic field strength configuration are intrinsically linked. We believe that stellar flares are caused by the reconnection of surface magnetic field loops (see, e.g. Yokoyama \& Shibata 1998 and reference therein). The correlation between magnetic field in active regions is very clear in the case of solar flares (Fletcher et al. 2011) meaning that stellar flares may be used as a proxy for magnetic activity.

The fraction of stars showing $\mathrm{H}_{\alpha}$ in their emission (Delfosse et al. 1998) or frequent flares (Hawley \& Pettersen 1991; Schmidt et al. 2014; Hawley et al. 2014) increases with decreasing mass, mainly due to their longer rotational braking times (Delfosse et al. 1998; Barnes 2003; Delorme et al. 2011). The braking rates strongly depend on the spectral type (or mass) of the star (Barnes 2003; Barnes \& Kim 2010), which results in partially convective low-mass stars spinning down slower than their higher-mass counterparts. Fully convective stars have even longer rotational braking timescales (Reiners \& Basri 2008; Browning et al. 2010). Low-mass $\mathrm{M}$ dwarfs exhibit stronger chromospheric and coronae emissions for a given rotation period than more massive G-K dwarfs (see Fig. 6 in Kiraga \& Stepien 2007). On the other hand, rotation and magnetic activity of stars decrease with 
time as a consequence of magnetic braking, that is the angular momentum is extracted from the convective envelope and lost through a magnetized wind (Skumanich 1972; Mestel 1984; Mestel \& Spruit 1987).

M dwarfs make up $70 \%$ of the stars in the solar neighbourhood. They are small, cool main sequence stars with temperatures in the range $2400-3800 \mathrm{~K}$ and radii between 0.10 and $0.63 R_{\odot}$. Radii of $\mathrm{M}$ dwarfs are smaller than solar-like stars, implying that the flare activity phenomena are more pronounced at the background of their cool photospheres. M dwarfs with spectral types later than M4 have fully convective atmospheres with no boundary zone between the radiative and convective zones (Hawley et al. 2014), implying that we do not expect significant magnetic fields such as those generated in the Sun (Doyle et al. 2018). The magnetic fields present at the surface of low-mass M dwarfs (M 5-M 8) originate either from strong, axisymmetric dipole fields or from weak, higher-order multipole fields (Morin et al. 2010, 2008).

Some of the first detailed optical observations of stellar flares on M dwarfs were reported by Bopp et al. (1973) and Gershberg \& Shakhovskaia (1983); see also Gershberg (2005). X-ray observations with the EXOSAT satellite detected the first flare in $\mathrm{M}$ dwarfs in the atmosphere of YZ CMi (Heise et al. 1975). Since then, the physics of stellar flares has been studied by different teams over a wide range of energy, from $\gamma$-ray to radio frequencies. The characterization of $\mathrm{M}$ dwarf flares has become possible thanks to the advent of large-scale surveys such as the Sloan Digital Sky Survey (SDSS; York et al. 2000). Kowalski et al. (2009) identified flares in the SDSS Stripe 82, where the authors found that active $\left(\mathrm{H}_{\alpha}\right.$ in emission) $\mathrm{M}$ dwarfs flared at approximately 30 times the rate of inactive $\mathrm{M}$ dwarfs. They also found that the flaring fraction increases for cooler stars, which they attribute in part to a higher fraction of magnetically active stars among late-M dwarfs. This is compatible with lower-mass stars rotating more rapidly over a long time-span (and therefore magnetically active) than their more massive counterparts. The presence of higher rates of flares in late-type $M$ dwarfs impacts the exoplanet community because flares release large amounts of energy in the UV, which could potentially affect the atmospheres and habitability of orbiting exoplanets.

Recent large-scale radial velocity (RV) searches for extrasolar planets (Jenkins et al. 2017, and references therein) provide a new framework and motivation to study stellar and flare activity phenomena. Indeed, they provide extensive time series of high-resolution spectra obtained by the best spectrographs on the biggest telescopes for large samples of stars. The study of activity in exoplanet science is of special interest because spots, plages, and other inhomogeneities of the stellar surface affect the shape of spectral lines, shift their centroids, and consequently bias the measured RV (e.g. Barnes et al. 2011, and reference therein). Stellar and flare activity produce additional signals usually handled as random noise (the so-called RV jitter), which should be added to other known systematics such as photon noise and instrumental instabilities (Astudillo-Defru et al. 2015; Suárez Mascareño et al. 2017).

Today, M dwarfs represent important targets for searches of exoplanets. Due to their low mass and luminosity, M dwarfs are ideal targets to find low-mass, Earth-size planets with the RV technique (see Gomes da Silva et al. 2012, and references therein) and look for possible transits. The first rocky planets around $\mathrm{M}$ dwarfs were detected by RV and transits (Rivera et al. 2015; Charbonneau et al. 2009). We note that the first rocky planet Corot-7b orbiting a G9V star was announced by the COROT team (Léger et al. 2009; Queloz et al. 2009).
Proxima Cen was recently highlighted as a planet-host mid-M dwarf (Anglada-Escudé et al. 2016). Proxima Cen b orbits its host star with a period of 11.2 days, corresponding to a semi-major axis distance of $0.05 \mathrm{AU}$, and has a mass close to that of the Earth (from 1.10 to 1.46 Earth masses) and orbits in the temperate zone (Anglada-Escudé et al. 2016).

Proxima Cen is a known flare star that exhibits random but significant increases in brightness due to magnetic activity (Christian et al. 2004). The spectrum of Proxima Cen contains numerous emission lines (Fuhrmeister et al. 2011; Pavlenko et al. 2017). These features most likely originate from plages, spots, or a combination of both. Pavlenko et al. (2017) found the presence of hot evaporated matter flow originating from the outer envelope. The atmosphere of Proxima Cen shows a rather complicated structure consisting of the cool photosphere, chromosphere, and hot outer layers where even lines of HeI form. In general, the flare rate of Proxima Cen is lower than that of other flare stars of similar spectral type, but is unusually high given its slow rotation period (Davenport et al. 2016). The star has an estimated rotation period of $\sim 83$ days and a magnetic cycle of about $7 \mathrm{yr}$ (Benedict et al. 1998; Suárez Mascareño et al. 2016; Wargelin et al. 2017). The X-ray coronal and chromospheric activity have been studied in detail by Fuhrmeister et al. (2011) and Wargelin et al. (2017). Recently, Thompson et al. (2017) claimed a detection of rotational modulation of emission lines in the spectrum of Proxima Cen.

West et al. (2015) suggested that the activity-rotation relation for the slow rotating stars may be more complicated than predicted by a simple spin-down model. One possibility could be that while persistent magnetic activity in late-type $\mathbf{M}$ dwarfs is rare (or non-existent) for slow rotators, the presence of magnetic cycles may produce observed activity in a small number of stars at any given time. As stated by Newton et al. (2016), many slowly rotating mid-to-late $\mathrm{M}$ dwarfs show variability amplitudes of half a per cent or more, implying that they have maintained strongenough magnetic fields to produce the requisite spot contrasts. The lack of correlation between rotation period and amplitude for these stars indicates that the spot contrast is not changing significantly, even when they undergo substantial spin-down.

To date, most of the rocky planets in the habitable zone have been found around these very low-mass stars (Udry et al. 2007; Bonfils et al. 2011; Quintana \& Barclay 2014; Torres et al. 2015; Wright et al. 2016; Anglada-Escudé et al. 2016). However, the flare activity of the hosting stars may affect the structure and temperature regime of the exoplanetary atmospheres, thereby affecting the size of the habitable zone. In March 2016, the Evryscope observed the first superflare visible to the naked eye detected from Proxima Cen (Howard et al. 2018). The brightness of Proxima Cen increased by a factor of 68 during this superflare and released a bolometric energy of $10^{33.5} \mathrm{erg}$, i.e. approximately ten times larger than any flare detected before. Modelling photochemical effects of $\mathrm{NO}_{x}$ atmospheric species generated by the severe particle events in the atmosphere of Proxima Cen b shows that the high level of the similar extreme strong flare activity in the long term is sufficient to reduce the ozone of an Earth-like atmosphere by $90 \%$ within five years. In other words, a conception of habitable zone should be reconsidered taking into account the impact of flare activity on exoplanet atmospheres (Howard et al. 2018).

In this paper, we focus on the temporal changes of emission lines in the optical spectra of Proxima Cen. We describe the observations in Sect. 2. We detail our procedure in Sect. 3. Section 4 presents our results, which we discuss and put into context in Sect. 5. 
Table 1. Emission lines studied in our paper.

\begin{tabular}{ccccc}
\hline \hline Line & $\lambda_{\text {air }}(\AA)$ & Transition & $E_{1}(\mathrm{eV})$ & Formation regions \\
\hline $\mathrm{H}_{\alpha}$ & 6562.81 & $2 \mathrm{P}^{0}-3 \mathrm{~S}, \mathrm{D}$ & 10.199 & Flares, facules \\
$\mathrm{CaII}(\mathrm{K})$ & 3933.66 & $4^{2} \mathrm{~S}_{1 / 2}-4^{2} \mathrm{P}_{1 / 2}^{0}$ & 0.0 & Chromosphere \\
$\mathrm{CaII}(\mathrm{H})$ & 3968.47 & $4^{2} \mathrm{~S}_{1 / 2}-4^{2} \mathrm{P}_{3 / 2}^{0}$ & 0.0 & Chromosphere \\
$\mathrm{He}_{5876}$ & 5875.64 & $2^{3} \mathrm{P}_{0}-3^{3} \mathrm{D}$ & 20.964 & Flares, transition zone \\
$\mathrm{He}_{4026}$ & 4026.19 & $2^{3} \mathrm{P}_{0}-5^{3} \mathrm{D}$ & 20.964 & Flares, transition zone \\
$\mathrm{H}_{\epsilon}$ & 3970.72 & $2 \mathrm{P}^{0}-7 \mathrm{~S}, \mathrm{D}$ & 10.199 & Flares \\
$\mathrm{NaI}\left(\mathrm{D}_{1}\right)$ & 5889.95 & $3^{2} \mathrm{~S}_{1 / 2}-3^{2} \mathrm{P}_{3 / 2}^{0}$ & 0.0 & Chromosphere \\
$\mathrm{NaI}\left(\mathrm{D}_{2}\right)$ & $5895 / 92$ & $3^{2} \mathrm{~S}_{1 / 2}-3^{2} \mathrm{P}_{3 / 2}^{0}$ & 0.0 & Chromosphere \\
\hline
\end{tabular}

\section{Optical spectra of Proxima obtained with 3.6-m/HARPS}

We analysed 386 optical spectra obtained with HARPS, a fibrefed, echelle, high-resolution spectrograph installed on the 3.6-m European Southern Observatory (ESO) telescope in the La Silla Observatory, Chile. The instrument offers a resolving power of $R \sim 115000$ over a spectral range from 3780 to $6810 \AA$. The dates cover a time interval of over $13 \mathrm{yr}$, from Barycentric Julian dates (BJD) between [245]3152.600, that is the night of May 27, 2004, and [245]6418.643, the night of September 30, 2017. In the remainder of the paper, we omit the prefix [245]. Most observations include one or two exposures per night, with some extended sets of exposures taken every $\sim 10 \mathrm{~min}$ at dates $\mathrm{BJD}=6417$ (May 4, 2013; number of exposures on that night is $N_{\mathrm{t}}=55$ ), 6418 (May 5, 2013; $N_{\mathrm{t}}=64$ ), 6420 (May 7, 2013; $N_{\mathrm{t}}=22$ ), and 6426 (May 13, 2013; $N_{\mathrm{t}}=9$ ). The HARPS observations cover an extended time interval providing a unique set of data to analyse the behaviour of stellar flares with time over a long period.

\section{Procedure}

We discuss the temporal changes of a few emission lines listed in Table 1. These lines result from different excitation potentials. Their formation requires different physical conditions, which occur in distant parts of the active atmosphere of Proxima Cen. Changes in the $\mathrm{pEW}$ and line profiles of these emission lines reflect the direct or indirect impact of the flare activity on the whole stellar atmosphere, as well as their changes in time.

We re-normalised the final 1D observed spectra to get the same flux in the background of these emission lines. This allows us to investigate the relative changes of $\mathrm{pEW}$ of emission lines with time. As shown in Pavlenko et al. (2017), the changes of the background of emission lines appear rather marginal. In other words, activity processes do not affect photospheric levels, where the most absorption features and continuum form.

We measured pEW of all emission lines listed in Table 1. Here we note that all these emission lines in the spectrum of Proxima Cen were analysed on the same spectra and, thus, at the same times. To investigate the temporal correlation between different emission lines formed in the flare events, we computed the Pearson $k_{\mathrm{p}}$ and Stockman $k_{\mathrm{s}}$ rank correlation coefficients to evaluate the changes in $\mathrm{pEW}$ of our emission lines versus $\mathrm{H}_{\alpha}$ :

$$
\begin{aligned}
& k_{\mathrm{p}}=\frac{n \sum x_{i} y_{i}-\sum x_{i} \sum y_{i}}{\sqrt{n \sum x_{i}^{2}-\left(\sum x_{i}\right)^{2}} \sqrt{n \sum y_{i}^{2}-\left(\sum y_{i}\right)^{2}}}, \\
& k_{\mathrm{s}}=\frac{\sum\left(R\left(x_{i}\right)-(n+1) / 2\right) \times\left(R\left(y_{i}\right)-(n+1) / 2\right)}{\left.\left.\left(\sum\left(R\left(x_{i}\right)-(n+1) / 2\right)\right)^{2} \times \sum\left(R\left(y_{i}\right)-(n+1) / 2\right)\right)^{2}\right)^{0.5}} .
\end{aligned}
$$

These coefficients change in the range $-1 \leq r_{x y} \leq 1$. By definition, the cases $k_{\mathrm{p}}, k_{\mathrm{s}}=1,0,-1$ correspond to the strong correlation, no correlation, and strong anti-correlation, respectively.

\section{Results}

The variability of the pEW of the selected emission lines on different dates is depicted in Fig. 1. These pEW were determined for one dataset of the observed spectra, see Table A.1. We draw our attention to a few expositions marked with the letters A, B, $\mathrm{C}, \mathrm{D}, \mathrm{E}$, and F:

- The time mark "A" locates the minimum level of activity of Proxima Cen, determined by the lowest $\mathrm{pEW}$ of $\mathrm{H}_{\alpha}$ (Sect. 4.4).

- The letters B, C, and D mark three flare events during observations on $\mathrm{BJD}=6417$ and 6418 (Sect. 4.5).

- The letters F and E mark two peaks of the "strong" flare seen in $\mathrm{H}_{\alpha}$ at $\mathrm{BJD}=6414$ (Sect. 4.8).

\subsection{Long-term variatons of $\min H_{\epsilon}$}

The temporal changes of the strength of the $\mathrm{H}_{\epsilon}$ line provide some evidence about the presence of long-term variability in Proxima Cen. The emission in $\mathrm{H}_{\epsilon}$ is weaker in comparison with $\mathrm{H}_{\alpha}$, still its relative intensity is more sensitive to the level of activity in comparison with other lines. We derive a quasi-periodic variability of $\sim 8200$ days by approximating the minimum values of the observed $\mathrm{pEW}$ of $\mathrm{H}_{\epsilon}$ at the dates BJD $=3000-8000$ by the relation $4 \times \sin (2 \times \pi(\mathrm{BJD}-2500) / 8200)$.

\subsection{Temporal changes of the emission lines}

We draw our attention to the temporal variability of emission lines in the spectrum of Proxima Cen. We argue that if all lines are formed in a single flare region of high temperature, we should see a strong correlation in their behaviours.

For simplicity, we adopt that the level of activity of Proxima Cen corresponds to the $\mathrm{pEW}$ of $\mathrm{H}_{\alpha}$ measured at a given date, that is, the minimum activity level of Proxima Cen corresponds to the minimum of the $\mathrm{pEW}$ of $\mathrm{H}_{\alpha}$ and vice versa. Indeed, the formation of $\mathrm{H}_{\alpha}$ seen in emission is possible only in high-temperature regions heated by the flare events.

To analyse the relative temporal behaviour of $\mathrm{H}_{\alpha}$ and other lines we computed the Pearson and Stockman correlation coefficients for each pEW (Table 3). We note that the spectra observed between BJD $=6417$ and 6429 were obtained with the Fabry-Perot wavelength calibration which generates some flux distortion at the exact position of the HeI at $4206 \AA$. Therefore, we excluded this line from our analysis for those dates.

In Table 3, we show the result of computations of the Pearson and Stockman correlation coefficients of the emission line 

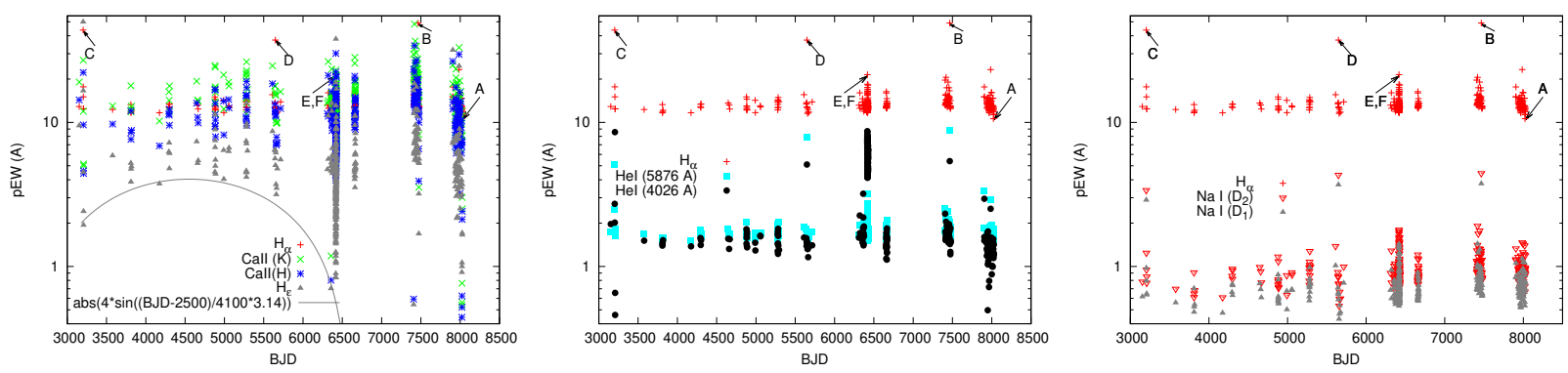

Fig. 1. Variations of pEW of emission lines at all dates of observations. The letter "A" marks the absolute minimum of the $\mathrm{pEW}$ of $\mathrm{H}_{\alpha}(\mathrm{Sect} .4 .4)$, letters "B", "C" and "D" label the flares occasionally observed at BJD =7465.8823, 3202.5647, and 5647.7300, respectively (Sect. 4.4) and letters "E" and "F" label the pEW of two $\mathrm{H}_{\alpha}$ flares observed across BJD=6417 (Sect. 4.2). Grey line shows the approximation of the set of minimum values of pEW of $\mathrm{H}_{\epsilon}$ by a sinusoid; see Sect. 4.1.

Table 2. Classification of the strength of flares adopted in this paper.

\begin{tabular}{cc}
\hline \hline Classification & $\mathrm{pEW}\left(\mathrm{H}_{\epsilon}\right)$ \\
\hline $0_{\epsilon}$ & $0 \mathrm{pEW}\left(\mathrm{H}_{\epsilon}\right)<2 \AA$ \\
$1_{\epsilon}$ & $2<\mathrm{pEW}\left(\mathrm{H}_{\epsilon}\right)<4 \AA$ \\
$2_{\epsilon}$ & $4<\mathrm{pEW}\left(\mathrm{H}_{\epsilon}\right)<16 \AA$ \\
$3_{\epsilon}$ & $\mathrm{pEW}\left(\mathrm{H}_{\epsilon}\right)>16 \AA$ \\
\hline
\end{tabular}

strength with $\mathrm{H}_{\alpha}$ for different time intervals. This was carried out (1) for all dates covered our interval of observations, from 3152.6001 to 6418.6431 , except for $\mathrm{BJD}=6417$ and 6429 in the case of the $\mathrm{He}_{4026}$ line, and (2) for two extended continuous subsets of observations of our lines except $\mathrm{He}_{4026}$ on $\mathrm{BJD}=6417$ and 6418 (Sect. 4.8).

In all cases we derive high positive values for the two correlation coefficients. In other words, we see that flare processes modulate the strength of all lines. Indeed, all lines follow the changes in intensity of $\mathrm{H}_{\alpha}$. The $\mathrm{He}_{4026}$ line shows the weakest correlation with $\mathrm{H}_{\alpha}$. We interpret this correlation as evidence that all lines are formed in or are affected by the same flare events which covered the broad range of heights in the stellar atmosphere.

In Fig. 11, we show the response of other lines on changes in the $\mathrm{pEW}$ of $\mathrm{H}_{\alpha}$. In fact, we can see three groups of lines with different correlation with $\mathrm{H}_{\alpha}$, which can be roughly characterised by the gradient $b=\mathrm{d}(\mathrm{pEW}) / \mathrm{d}\left(\mathrm{pEW}\left(\mathrm{H}_{\alpha}\right)\right)$. CaII $\mathrm{H}, \mathrm{K}$, and $\mathrm{H}_{\epsilon}$ show larger $b$, while both helium lines show the weakest dependence; the relative response of $\mathrm{NaI}$ is stronger than $\mathrm{He}_{4026}$, but weaker than CaII $\mathrm{H}$ and $\mathrm{K}$.

For convenience we implement a rather phenomenological classification of the strength of flares using quantitative criteria based on the values of the $\mathrm{pEW}$ of $\mathrm{H}_{\epsilon}$ (Table 2; Fig. 11).

We favour the use of $\mathrm{H}_{\epsilon}$ to classify flares because its $\mathrm{pEW}$ is strongly sensitive to the strength of flares. At the minimum of the flare activity, this line almost disappears (see Sect. 4.4). At maximum activity, the $\mathrm{pEW}$ of $\mathrm{H}_{\epsilon}$ might even be higher than the $\mathrm{pEW}\left(\mathrm{H}_{\alpha}\right)$, see Fig. 11. However, this is only true for $\mathrm{pEW}$ computed with respect to the local pseudo-continuum, which can itself change during the flares (Sect. 4.6). Nonetheless, the equivalent widths of $\mathrm{H}_{\alpha}$ always exceed the equivalent widths of $\mathrm{H}_{\epsilon}$ due to the exponential drop of the flux towards the blue part of the spectrum.

\subsection{Photosphere, chromosphere, and flare regions}

In this paper, we discuss different aspects of the flare activity of Proxima Cen. For simplicity, we adopt that the atmosphere of Proxima Cen consists of three parts.
- The photosphere is the deepest part of the atmosphere, in which molecular and atomic absorption spectrum of Proxima. The photosphere of Proxima seems to be stable; flares do not affect its structure, because molecular lines are practically not affected by the flare events.

- The chromosphere lies above the photosphere and the temperature increases outwards. Here the formation of most emission lines occurs in NLTE. The chromosphere is a quasi-stable structure heated by the dissipation of non-thermal flows from the convective photosphere. Chromospheric lines are comparatively narrow due to absence of large-scale moves.

- According to modern notions, the flares are located in the outer part of a chromosphere and/or in the corona. Again, for simplicity we adopt flare conception described in Yokoyama \& Shibata (1998). Just after an avalanche-like process of the release of energy due to the re-connection of magnetic field loops a small volume of the atmosphere of Proxima Cen (up to a few percent of the surface in the case of strongest flares) is heated up to $10^{6} \mathrm{~K}$ or more. Afterwards, the upward flows of hot matter from chromosphere (evaporation) and downward flows of cooling matter (condensation) toward photosphere are formed. Due to the higher temperatures in the flare regions, thermal and nonthermal motions are much higher and emission lines are broader than chromospheric lines. We follow this simplified model to avoid the uncertainty of interpretation of our observed data due to different conceptions based on the numerous theoretical models. In reality, a flare is a multi-dimensional magnetohydrodynamic process, which is variable on short timescales. Current models of flares are much more complicated (Zhang et al. 2016, and references therein); the analysis of which is beyond the scope of this paper.

\subsection{Emission lines at the minimum activity of Proxima Cen}

The photospheric spectrum at least in the optical and nearinfrared spectral region shows rather marginal response to the flares (Pavlenko et al. 2017). On the contrary, both profiles and intensities of our emission lines respond to the changes of activity; see Fig. 1.

Firstly, we find the three lowest $\mathrm{pEW}$ of $\mathrm{H}_{\alpha}$ in the spectrum of Proxima Cen, with pEW of 10.62, 11.34, and $11.39 \AA$ at dates $\mathrm{BJD}=8021.4600,8023.4639$, and 8027.4785 , respectively. The exposure taken on BJD $=8021.4600$ is marked by "A" in Fig. 1 . These three dates differ by only a few days and show a minimum activity level of Proxima Cen.

The left-hand side panel of Fig. 2 compares the level of activity of the selected lines on those three dates of minimum activity of Proxima Cen. The right-hand side panel of Fig. 2 shows the 
Table 3. Pearson and Stockman correlation coefficients of the computed pEW of the emission lines considered in this work vs. the $\mathrm{pEW}$ of $\mathrm{H}_{\alpha}$.

\begin{tabular}{ccccccc}
\hline \hline & \multicolumn{2}{c}{$\mathrm{All}, N=386$} & \multicolumn{2}{c}{$6417^{d}, N=55$} & \multicolumn{2}{c}{$6418^{d}, N=23$} \\
\hline $\mathrm{H}_{\alpha}$ vs. line & $k_{\mathrm{p}}$ & $k_{\mathrm{s}}$ & $k_{\mathrm{p}}$ & $k_{\mathrm{s}}$ & $k_{\mathrm{p}}$ & $k_{\mathrm{s}}$ \\
\hline $\mathrm{CaII}(\mathrm{K})$ & 0.8045 & 0.7229 & 0.9531 & 0.7832 & 0.8042 & 0.7955 \\
$\mathrm{CaII}(\mathrm{H})$ & 0.8897 & 0.7847 & 0.9753 & 0.8188 & 0.8068 & 0.8098 \\
$\mathrm{He}_{5876}$ & 0.9288 & 0.8389 & 0.9377 & 0.7286 & 0.6407 & 0.6055 \\
$\mathrm{He}_{4026}$ & 0.5265 & 0.4420 & & & & \\
$\mathrm{H}_{\epsilon}$ & 0.9068 & 0.7506 & 0.9210 & 0.8353 & 0.5907 & 0.6118 \\
$\mathrm{NaI}\left(\mathrm{D}_{2}\right)$ & 0.9397 & 0.8824 & 0.9456 & 0.7877 & 0.9673 & 0.9555 \\
$\mathrm{NaI}\left(\mathrm{D}_{1}\right)$ & 0.9496 & 0.8832 & 0.9489 & 0.8108 & 0.9628 & 0.9617 \\
\hline
\end{tabular}

Notes. Dates between BJD = 6417 and 6429 were not accounted for $\mathrm{He}_{4026}$ line (see Sect. 4.2).

relative change of these lines between the times of maximum and minimum activity.

At times of minimum activity, we find the following.

- We still see an emission component in the blue wing of $\mathrm{H}_{\alpha}$ with $V=-30 \mathrm{~km} \mathrm{~s}^{-1}$ (Pavlenko et al. 2017), formed by the rapidly moving evaporating plasma, see Abbett \& Hawley (1999). In the core of the $\mathrm{H}_{\alpha}$ emission, we see a self-absorption feature formed by the flows of neutral hydrogen moving towards and away from the observer; see the top-left panel of Fig. 2.

- The interesting phenomenon is the almost complete disappearance of emission CaII $\mathrm{H}$ and $\mathrm{K}$ as well as emission of $\mathrm{H}_{\epsilon}$ (second plot from the top in the left panel of Fig. 2). Then, at the moment of the relatively stronger $\mathrm{H}_{\alpha}$ we see the weaker emission of both $\mathrm{CaII} \mathrm{H}$ and $\mathrm{K}$ lines.

- A notable difference in the $\mathrm{H}_{\epsilon}$ and CaII $\mathrm{H}$ lines in Fig. 2 (right panel) is the broadening. Paulson et al. (2006) discussed the broadening of Balmer lines formed during flare events in the Barnard star and noted some problems in the physical explanation of their observed widths. However, in our case the CaII lines and $\mathrm{H}_{\epsilon}$ form in different parts of the atmosphere of Proxima Cen. CaII $\mathrm{H}$ and $\mathrm{K}$ lines are collisionally controlled lines that form in the quasi-stable chromosphere whereas Balmer lines are lines controlled by the photoinisation; they form in the hightemperature flare region(s) where dispersion of thermal and/or non-thermal velocities is larger, generating broader emission lines (Thomas 1957).

- The emission in the cores of the $\mathrm{NaI}_{1}$ and $\mathrm{D}_{2}$ doublet correlates with the $\mathrm{H}_{\alpha}$ line (third left panel from the top of Fig. 2). The emission cores of $\mathrm{NaI} \mathrm{D}_{1}$ and $\mathrm{D}_{2}$ lines are still strong at minimum activity although two times weaker than at maximum activity (panels in third row from the top in Fig. 2). Furthermore, these emission lines at the minimum of flare activity are affected by the absorption of cool condensed matter moving outwards from the star. Indeed, blue parts of the emission lines are more affected by absorption. In other words, moving outwards cool condensed matter "eats" the blue part of the NaI emission lines.

- At the position of the HeI line at $4026 \AA$, we observe a narrow feature in the spectrum taken on $\mathrm{BJD}=8027.4785$ (fourth plot on the left panel of Fig. 2). However, the spectrum in the blue is noisier compared to other days. On the other hand, this "nebular-like" line is at least four pixels wide, and therefore it may be a real feature formed somewhere in the outer atmosphere of the star in specific circumstances. Unfortunately, we cannot investigate the temporal evolution of this feature to verify its origin.

- $\mathrm{He}_{5876}$-like emission is absent on the days of minimum activity (left bottom panel of Fig. 2). Paulson et al. (2006) noted that the $\mathrm{He}_{5876}$ line only appears during strong-enough stellar flares. In other words, the lack of $\mathrm{He}_{5876}$ at the minimum of activity corresponds to a low level of activity. We note that this line is located in the optical spectral region where observed photosphetic fluxes show little variation from quiet to flare modes; see Sect. 4.6.

The time interval of the minimum activity of Proxima Cen likely extends for a few days to a few weeks. In other words, at the times of the last observations made in 2017, we observe the star in the state of absolute minimum activity.

\subsection{Emission lines at the maximum activity of Proxima Cen}

During the maximum activity of Proxima Cen, we observe all our lines in emission (right-hand panel of Fig. 2). The three strongest flares are highlighted in Fig. 1 with the letters B, C, and D. All belong to the $3_{\epsilon}$ flares.

- During these strong flares, we see the fine structure of the self-absorption in the core of the strong $\mathrm{H}_{\alpha}$ emission line, which can be interpreted as evidence of small-scale neutral hydrogen flows in the outer part of the flares. The strongest $\mathrm{H}_{\alpha}$ profile shows a well-defined red asymmetry due to inward movement of hot matter (top-right panel of Fig. 2). We measure a velocity $V_{\mathrm{r}}$ of $150 \mathrm{~km} \mathrm{~s}^{-1}$ for the B flare, where $V_{\mathrm{r}}$ is the motion of the hot plasma from the flare regions.

- The strength of the CaII $\mathrm{K}$ and $\mathrm{H}$ emission lines anticorrelates with $\mathrm{H}_{\alpha}$. The strongest emission of $\mathrm{H}$ and $\mathrm{K}$ lines is observed at the same time as the "D" flare, the third strongest flare by intensity (second plot on the right panel of Fig. 2). Interestingly, we find the self-absorption in the cores of CaII $\mathrm{H}$ and $\mathrm{K}$ lines in all B, C, and D flares. The self-absorption is most likely formed in the outer, colder condensed flows, containing a number of CaII ions.

- The strength of the emission of the $\mathrm{D}_{1}$ and $\mathrm{D}_{2} \mathrm{NaI}$ lines also anti-correlates with $\mathrm{H}_{\alpha}$ (third plot on the right panel of Fig. 2). However, the strongest flare (B) provides the extended wings with $V_{\mathrm{r}}$ up to $\pm 80 \mathrm{~km} \mathrm{~s}^{-1}$. In this case the formation region of the $\mathrm{H}$ and $\mathrm{K}$ line wings is likely affected by the strong turbulence coming from the flare region.

- The strong flares generate strong helium emission lines of $\mathrm{He}_{4026}$ and $\mathrm{He}_{5876}$ (fourth and bottom plots on the right-hand side of Fig. 2). We see that both lines are stronger when $\mathrm{H}_{\alpha}$ is stronger, although this correlation remains rather qualitative. The second strongest $\mathrm{H}_{\alpha}$ flare provides the weakest He lines (lower left-hand panels of Fig. 2). Again, in the case of $\mathrm{He}_{5876}$ line, we witness an extended red wing up to $V_{\mathrm{r}}=100 \mathrm{~km} \mathrm{~s}^{-1}$, likely due to the infalling hot matter on the star. 

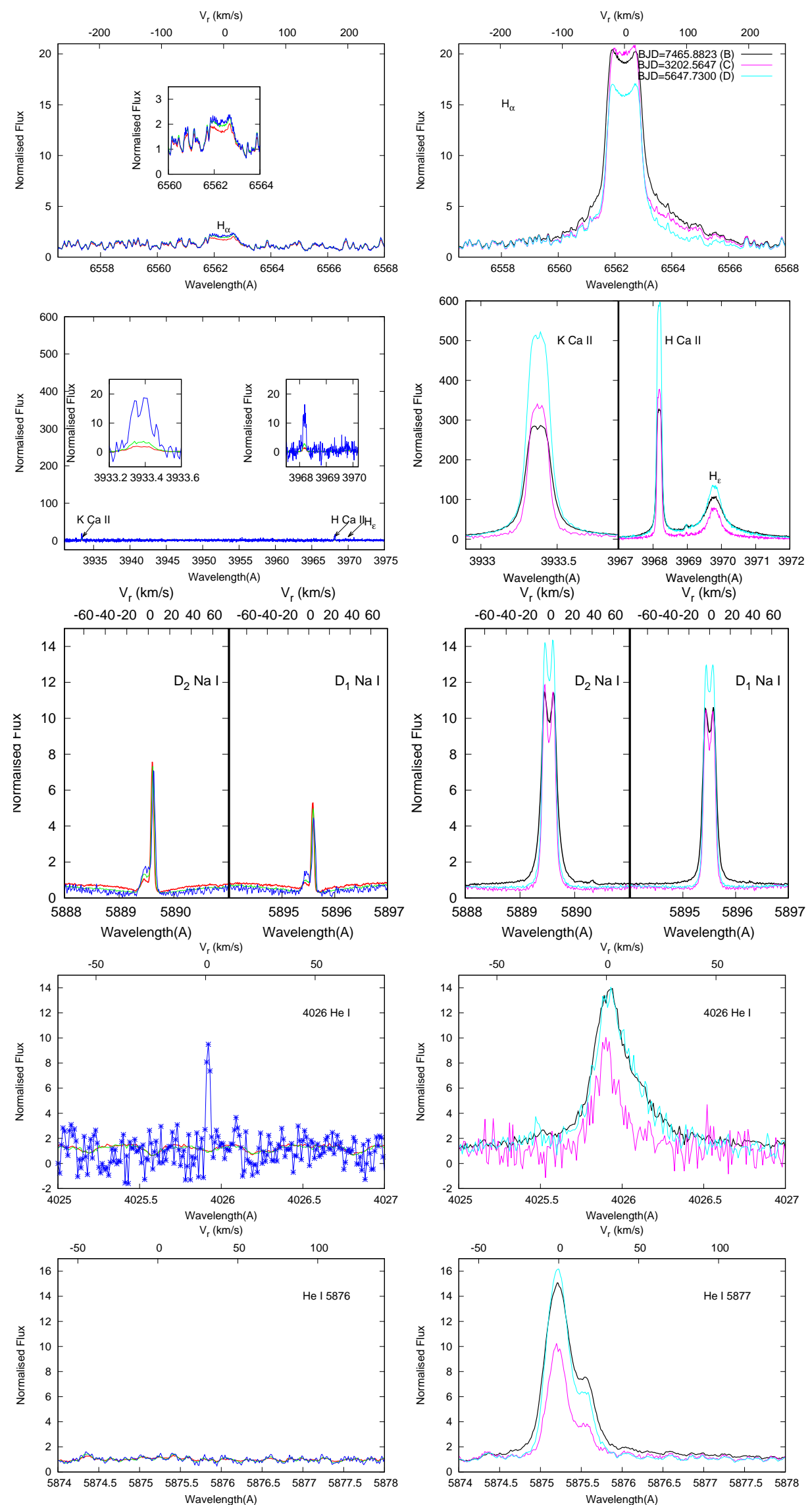

Fig. 2. Left panels: profiles of several emission lines, including $\mathrm{H}_{\alpha}$, CaII $\mathrm{K}$ and $\mathrm{H}, \mathrm{NaI}_{2}$ and $\mathrm{D}_{1}, \mathrm{He}_{5876}$, and $\mathrm{He}_{4026}$ at the three dates of minimum activity of Proxima Cen: BJD=8021.4600 (red), 8023.4639 (green), and 8027.4785 (blue). These dates are marked with letter "A" in Fig. 1. Right panels: same lines at the dates of maximum of activity of Proxima Cen, i.e. BDJ =7465.8823 (B; black), 3202.5647 (C; cyan), 5647.7300 (D; magenta) displayed in Fig. 1. 

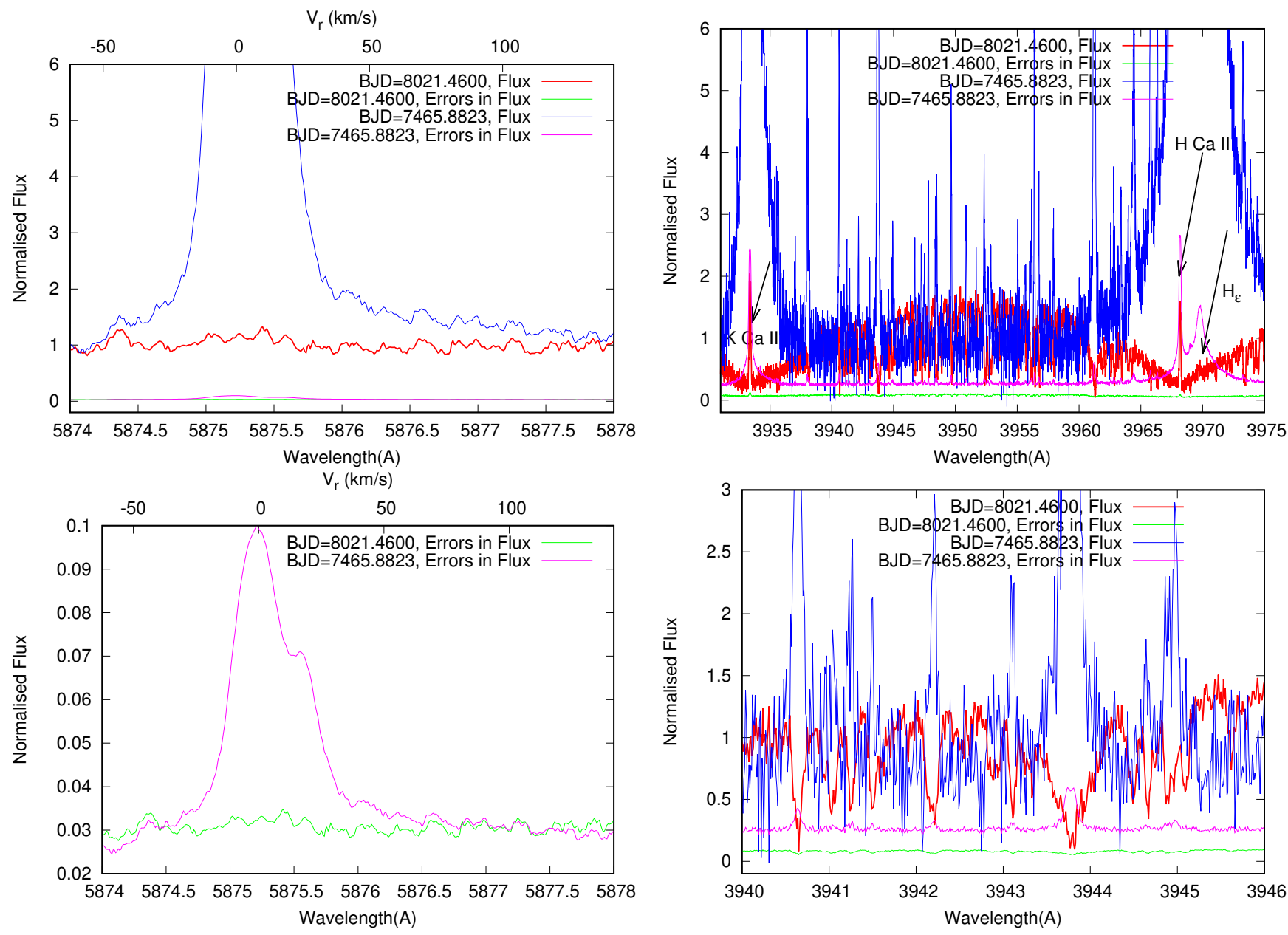

Fig. 3. Top-left panel: observed fluxes of emission lines across $\mathrm{He}_{5876}$ spectral region at the dates of minimum and maximum activity, shown by red and blue lines, respectively. Uncertainties of the flux determination are shown by the green and pink colours, respectively. Bottom-left panel: uncertainties of the flux determinations at the phases of minimum and maximum activity shown in larger scale. Top-right panel: observed fluxes of emission lines across CaII $\mathrm{H}$ and $\mathrm{K}$ and $\mathrm{H}_{\epsilon}$ spectral regions at the same dates of minimum and maximum activity, shown by red and blue lines, respectively. Bottom-right panel: part of the observed spectra shown in larger scale. Flux measurement uncertainties are also shown.

\section{6. $\mathrm{S} / \mathrm{N}$ and accuracy of the $p E W$ measurements}

We analyse spectra of Proxima Cen obtained by different authors on different dates. One of the key questions is whether or not our measurements are reliable, and therefore whether or not we can compare the $\mathrm{pEW}$ obtained on different dates during different levels of activity. To answer this question, we compared spectra observed in the blue $(\lambda<4200 \AA)$ and optical (4200 $⿱ 亠 0 .<<8000 \AA)$ spectral regions at different levels of Proxima activity. The flux of $\mathrm{M}$ dwarfs drops towards the blue spectral region, and therefore the quality of the observed spectra in the blue and optical regions may differ simply due to "natural" reasons. Furthermore, the blue-end of the spectrum is more sensitive to changes in the physical properties of emitted matter.

In Fig. 3 we show optical (left panel) and blue (right panel) wavelength ranges of the spectra of Proxima obtained on the dates of minimum $(\mathrm{BJD}=8021.46)$ and maximum activity $(\mathrm{BJD}=7465.8823)$. The optical and blue spectral ranges contain $\mathrm{He}_{5876}$ and $\mathrm{CaII} \mathrm{H}$ and $\mathrm{K}$ emission lines, respectively. We emphasise that we work with the residual fluxes, that is fluxes reduced to the level of the local pseudocontinuum by 1.0. Due the presence of molecular absorptions in the optical spectral range, the true continuum is buried under the absorption features formed by the numerous strong molecular bands. To the contrary, the continuum is still seen in the blue spectral region, which is more or less free from molecular absorption (Pavlenko et al. 2017). The local pseudocontinuum in this part of the spectrum is formed by the wings of strong atomic lines seen in absorption and/or in emission.

In Fig. 3 we show the estimations of the accuracy of flux measurements, $F_{\mathrm{a}}$, provided by the HARPS pipeline. In the first approach we can adopt $F_{\mathrm{a}} / F \approx S / N$, where $F$ is the monochromatic flux.

Accuracy of the flux measurements in the optical spectral region seems to be similar in the flare and quiet modes; see left panels of Fig. 3. Furthermore, in the quiet mode the S/N is similar for the observed blue and optical spectra.

On the other hand, in the case of the CaII $\mathrm{H}, \mathrm{K}$, and $\mathrm{H}_{\epsilon}$ lines, we see different $\mathrm{S} / \mathrm{N}$ for the spectra obtained in the flare and quiet modes. In the flare mode we observe a decrease in $\mathrm{S} / \mathrm{N}$ from $\sim 10$ to $\sim 3$ (see the top-right panel of Fig. 3), which cannot be explained by instrumental effects, such as different seeing or different exposures, because observations in the optical spectral regions provide the same quality, that is $\mathrm{S} / \mathrm{Ns}$ in the flare and quiet modes are similar. Numerous emission lines appear in the flare mode. At the corresponding wavelengths we see absorption lines in the quiet mode, as shown in the bottom-right panel of 
Fig. 3. Nevertheless, we still clearly see the drop in $\mathrm{S} / \mathrm{N}$ for the blue spectra obtained in the flare mode.

Likely, something happens with absolute fluxes in the blue spectral region. Namely, a decrease in $\mathrm{S} / \mathrm{N}$ may be explained in the case of the drop of these fluxes in the blue spectral region during the flares. In turn, any decrease in flux may be caused by an increase in opacity in the photospheric layers due to the non-thermal ionisation of metals (and/or hydrogen?), which are the main donors of free electrons in photospheres of cool stars. Over-ionisation of metals (and/or hydrogen?) increases the number density of $\mathrm{H}^{-}$, which is a very important source of opacity in stellar atmospheres.

On the other hand, we do not see any significant differences in $\mathrm{S} / \mathrm{N}$ in the optical spectra obtained in quiet and flare modes; this could be interpreted as a very weak dependence of the observed fluxes here on the flare activity.

Detailed analysis of these phenomena is beyond the scope of this paper. We simply note that despite the possible drop in flux in the blue spectral region, the level of the pseudocontinuum can be determined with sufficient accuracy during the quiet phase, but lower accuracy during flares. However, in the last case the strong emission cores are the main contributors to the $\mathrm{pEW}$. The emission cores can be measured with higher accuracy due to larger fluxes and higher S/N. We observe the opposite effect for weak emission lines in the quiet mode: both the pseudocontinuum and $\mathrm{pEW}$ are well determined when $\mathrm{S} / \mathrm{N}$ is greater than 10

Overall, we estimate an error budget of less than $10 \%$ of the $\mathrm{pEW}$ after taking into account all effects that may affect the quality of the spectra in the quiet and emission phases. We reiterate that $\mathrm{pEW}$ measurements are done with respect to the local pseudocontinuum, which itself can change depending on physical processes in and/or over its formation levels.

Fuhrmeister et al. (2011) reported activity in Proxima Cen on $\mathrm{BJD}=4901$, 4902, and 4903, that is March 10-12, 2009. On the third night, these latter authors observed a strong flare from X-ray to the optical, and UV excess (see their Fig. 13). They also observed many strong lines in the blue spectral range, including FeII, SiII, and TiII in the 3200-4200 A region. Likely, Fuhrmeister et al. (2011) observed an even stronger flare, known as a white flare, a phenomenon known in solar and stellar astrophysics (Walkowicz et al. 2011; Davenport et al. 2014, and references therein). In Fig. 11 of Fuhrmeister et al. (2011) we see that the $\mathrm{S} / \mathrm{N}$ in their UVES spectra is similar during the quiet and flare modes, suggesting that the increase of the flux in the blue spectral region does not affect the $\mathrm{S} / \mathrm{N}$ in the observed spectra. This effect is contrary to our case, where the $\mathrm{S} / \mathrm{N}$ increases during flare events. Unfortunately, the dates of Fuhrmeister et al. (2011) do not overlap with the dates of the HARPS database so we cannot carry out a direct comparison. Howard et al. (2018) also reported a white flare detected with the Euroscope telescope.

\subsection{Flare during the night of $B J D=6417$}

On the night of $\mathrm{BJD}=6417$ we find a flare of strength $3_{\epsilon}$ that was fully covered by uniform observations with time steps of 10 min until it recovered its "normal" state. Although $10 \mathrm{~min}$ might seem long for flare processes, we can investigate the main stages of this event. Moreover, we are able to simultaneously cover a significant wavelength range containing several emission lines discussed in this work.

On the night of $\mathrm{BJD}=6417$, we see a flare which started at 6417.6299 (labelled as " $\mathrm{E}_{1}$ " and corresponding to $t=-34.6 \mathrm{~min}$ ) and reached its maximum at 6417.65 (i.e. $t=0.0 \mathrm{~min}$, marked as "E" in the top-left panel of Fig. 4). We observe a temporal fading of that flare $\left(\mathrm{BJD}=6417.6841\right.$ or $t=+43.02 \mathrm{~min} ; \mathrm{F}_{1}$ ) and a secondary maximum (secondary flare?) of a strength of $2_{\epsilon}$ at $6417.69(t=+60.0 \mathrm{~min}$; "F") that later restored to normal state at 6417.7642 ( $t=160.00 \mathrm{~min}$, " $\mathrm{F}_{2}$ "). In Fig. 4, we show the time mark $\mathrm{E}_{2}(\mathrm{BJD}=6417.6445 t=-12.6 \mathrm{~min})$; at this time we see transit from a comparatively slow increase to an avalanchelike increase in flare intensity. The total duration of this flare is about $200 \mathrm{~min}$, i.e. more than $3 \mathrm{~h}$.

This flare is not the strongest in our observational set (Fig. 1). Although this phenomenon may differ from the other strong flares marked with letters "B", "C", and "D" in Fig. 1, we note a few interesting facts:

- The $\mathrm{H}_{\alpha}$ line shows a symmetrical profile (see top-left panel of Fig. 5), as in the case of flares C and D shown in Fig. 2.

- We see a good correlation between the strength of all lines and the strength of $\mathrm{H}_{\alpha}$. We interpret this as all lines most likely forming in one flare space.

- The cores of all lines are affected by the self-absorption, suggesting that we see multi-component structures of the absorbing condensed matter.

- The fine structure of the outer layers affects the cores of all lines shown in Fig. 5. This structure of the outer layers changes with time on short timescales. Indeed, we see differences in the cores of the lines observed at $t=+43.02$ and +60.0 mins displayed with pink and cyan lines, respectively, in Fig. 5. Even in the case of the CaII $\mathrm{K}$ line, we note a self-absorption component shifted by different $V_{\mathrm{r}}$ at different flare stages.

- We note the self-absorption components in the cores of the $\mathrm{CaII}$ and $\mathrm{He}_{5876}$ lines which are moving bluewards and redwards reflecting outward and inward flows of hot matter in the outer atmosphere, respectively (top-right and bottom-left panels of Fig. 5).

- The multi-component structure of the active regions of the atmosphere is also reflected in the shape of $\mathrm{D}_{1}$ and $\mathrm{D}_{2} \mathrm{NaI}$ lines. In the "quiet" state, we recognise the absorption components of the cores of emission lines. In the "active" phases the emission line intensity increases and self-absorption becomes notable; see the bottom-right panel of Fig. 5. However, this second emission core forms with another self-absorption component, which we interpret as at least two regions of line formation in the cases of $\mathrm{D}_{1}$ and $\mathrm{D}_{2}$.

\subsection{Flare activity on $B J D=6718,6420$, and 6426}

Other extended and well sampled sets of spectroscopic observations of the Proxima Cen were collected during the nights $\mathrm{BJD}=6718,6420$, and 6426. From the comparison of spectral lines in emission obtained at the same times, we may study the physics of the flare variability over short timescales thanks to exposures taken every $\sim 10 \mathrm{~min}$. Hence, we can investigate the relative responses of different lines during flare events.

During those nights, we detect a few flare events covered by exposures made at varying time intervals. We show the changes of pEW of several lines during those nights in Fig. 4. For convenience, the upper $x$-axis is given in minutes. Time $t=0 \mathrm{~min}$ corresponds to the maximum of the flares.

On $\mathrm{BJD}=6418$, we detect a flare in some lines with a duration of $250 \mathrm{~min}$ (top-right panel of Fig. 4). The strength of the flare is of order $2 \epsilon$. We most likely capture part of the flare, which we explain from the weak changes in amplitude of the emission lines and the absence of reaction of the $\mathrm{He}_{5876}$ line. On the other 

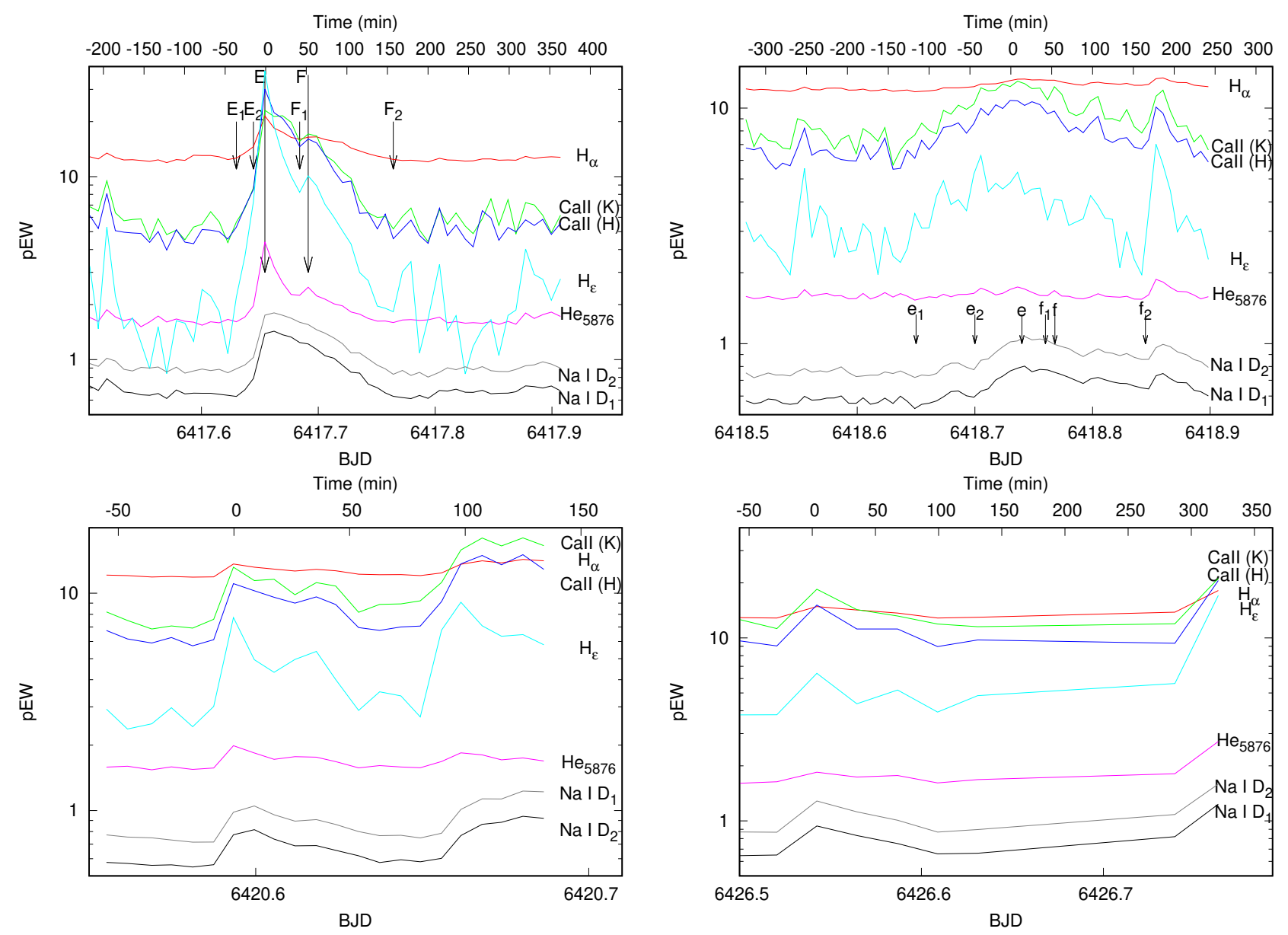

Fig. 4. Temporal variations of the pEW of several emission lines during four nights on $B J D=6417,6418,6420$, and 6426. Time marks with small letters depict the phases of the flare on 6418 corresponding to the same phases as on BJD $=6417$.

hand, we see the same flare phases, marked with small letters, as on the night of 6417 .

On BJD $=6420$ and 6426 , we detect a flare event of $100 \mathrm{~min}$ in duration with a strength of $2_{\epsilon}$ (bottom-left and bottom-right panels of Fig. 4). Again, we see the secondary flares at $t=+40$ or +50 min. Interestingly, on $\mathrm{BJD}=6426$ we see a flare "extended in time", where the strength of $\mathrm{H}_{\epsilon}$ corresponds to the $2_{\epsilon}$ flare.

It is worth noting again that the behaviour of $\mathrm{H}_{\epsilon}$ is more sensitive to the flare events compared with other lines. We suggest that this line is formed mostly in the active region of high temperature. We observe strong changes of the $\mathrm{pEW}$ of $\mathrm{H}_{\epsilon}$; even in the case of the extended flare on $\mathrm{BJD}=6418$, where changes of $\mathrm{H}_{\alpha}$ and other lines are weaker than on the previous night (top-left panel of Fig. 4).

\subsection{Rotational modulation of the chromosphere and the photosphere}

Here, we aim at detecting the rotation of the chromosphere of Proxima Cen thanks to the extended sets of HARPS highresolution spectra. We removed the two flare events and the deviant data points from the $\mathrm{pEW}$ dataset of the CaII $\mathrm{K}$ line, and subtracted the median values per observing epoch. Otherwise, it is not possible to see the modulation, if it exists, because of the different amplitudes of the line intensity at the different epochs and activity states. The Lomb-Scargle periodogram analysis (Lomb 1976; Scargle 1982) yields no significant strong peak. This is probably because of the artificial noise introduced by the removal of the median values from the original data. However, in the interval 30-1100 days, the highest peak lies at 90.72 days (with an uncertainty of \pm 1.5 days as derived from the FWHM of the peak). The second-highest peak of the periodogram lies at $81.68 \pm 1.5$ days. We note however that the periodogram is rather sensitive to the removal of deviant data. Figure 6 displays the median-subtracted CaII $\mathrm{K}$ pEW folded in phase with the period of 90.72 days. The data show a sinusoidal modulation with a small amplitude of $1.5 \AA$. A similar sinusoidal pattern is found when using the 81.68-day period, thus making the two periodicities indistinguishable with current HARPS spectra. The two values differ from the rotational period of $116.6 \pm$ 0.7 days determined from a subset of 222 HARPS spectra included here by Suárez Mascareño et al. (2015), but are closer to the period of $\sim 83$ days reported by Benedict et al. (1998), Kiraga \& Stepien (2007), and Wargelin et al. (2017). These latter authors used photometric observations obtained with the Hubble Space Telescope (HST) and the automated sky survey ASAS (Pojmanski 1997; Pojmanski \& Maciejewski 2004). A periodicity of $82.6 \pm 0.1$ days is also reported by Collins et al. (2017) after combining UVES, HARPS, and ASAS photometry. These authors discussed that the $116.6^{d}$ measurement published by Suárez Mascareño et al. (2015) is likely an alias induced by the specific HARPS observation times, and concluded that the most significant rotation period of Proxima Cen is $82.6 \pm 0.1$ days.

In the recent seventh data release (DR7) of the MEarth project (Berta et al. 2012; Irwin et al. 2011), Proxima Cen is included with more than 64200 photometric magnitudes obtained with the RG715 filter between 2014 May and 2017 August. Irwin et al. (2011) provided a detailed review on the 

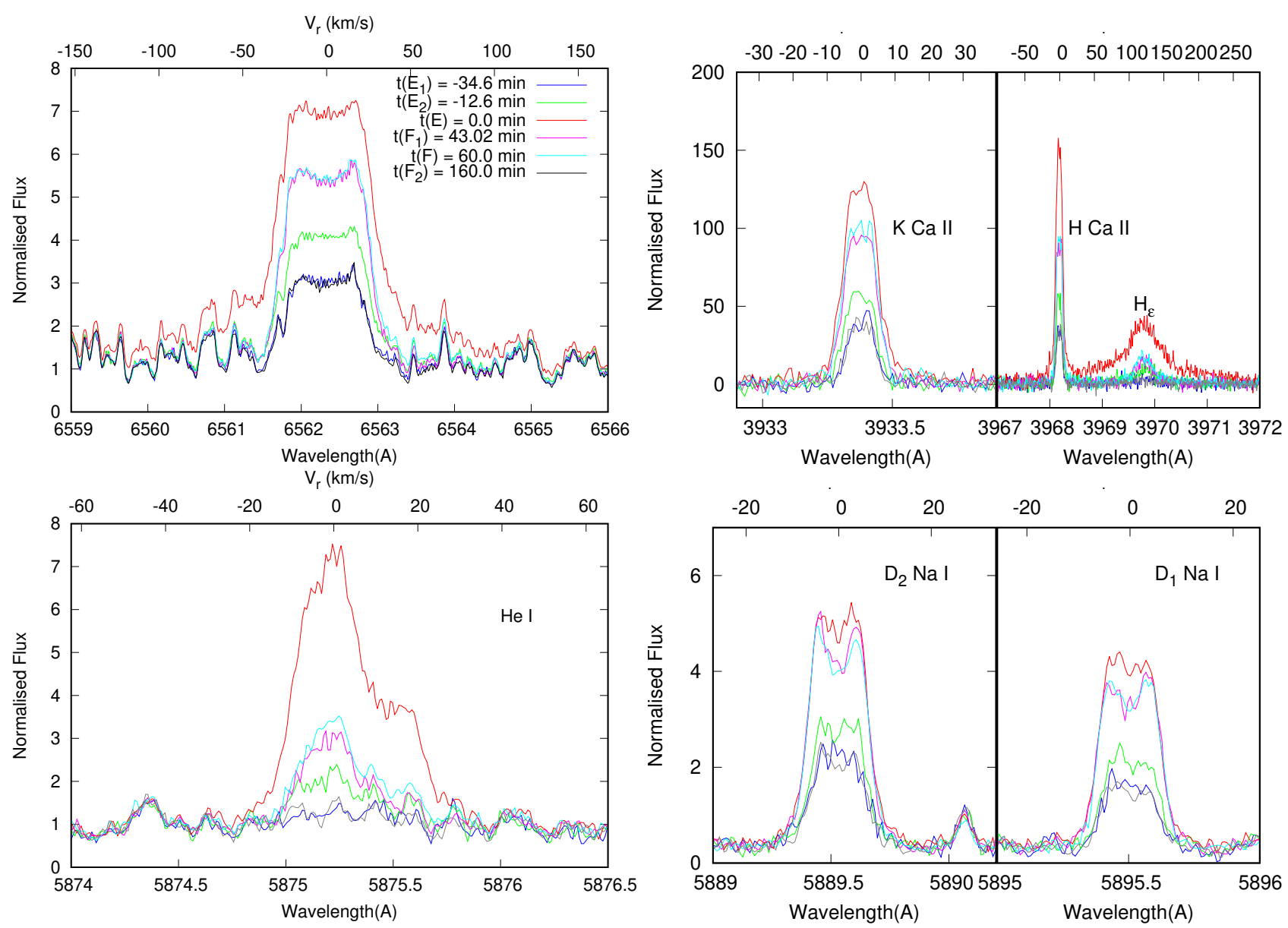

Fig. 5. Temporal variations of the $\mathrm{pEW}$ of several emission lines during the flare on the night of $\mathrm{BJD}=6417$. The times depicting the different phases are marked at the top of the top-left panel showing changes in the strength of $\mathrm{H}_{\alpha}$.

observing cadence, exposure times, and processing of the enormous amount of data of the MEarth monitoring campaigns. Concerning Proxima Cen, the resulting MEarth photometric light curve is illustrated in the top panel of Fig. 7, where the data present a dispersion of $\pm 30 \mathrm{mmag}$, which is three times larger than the typical photometric error bar. A quick inspection of the light curve reveals some sinusoidal patterns with small amplitudes at various epochs (e.g. BJD $=2457400)$. After applying a $2-\sigma$ clipping algorithm to remove the most deviant data points, we obtained the Lomb-Scargle periodogram of the MEarth DR7 light curve shown in the top panel of Fig. 8. A strong peak is detected at $91.0 \pm 3.0$ days (a second strong peak is seen at $\approx 124$ days, but it has a non-negligible contribution from an alias of the 91.0-day peak. This is most likely an alias of the annual window, i.e. $\left.P_{\text {alias }}=1 /(1 / 91-1 / 365)=121^{d}\right)$. Interestingly, the MEarth DR7 periodogram shows quite a number of peaks well above the false-alarm probability (FAP) of $0.1 \%$, suggesting that they may be significant frequencies of the data. We removed the strong 91.0-day periodicity from the original photometry, and a forest of significant peaks still remain in the periodogram. These peaks lie at 39.4, 42.9, 56.9, 83.2, 116.6, 129.2, and 167.3 days (with estimated uncertainty of 1-3 days), and all have associated amplitudes between 3 and 7 mmag (smaller than the quoted amplitude of the 91.0-day peak; see below). We note that two of these frequencies, 83.2 and 116.6 days, are consistent at the $1-\sigma$ level with those previously reported in the literature using very different datasets (Benedict et al. 1998; Kiraga \& Stepien 2007; Suárez Mascareño et al. 2015; Wargelin et al. 2017; Collins et al. 2017). Therefore, there is little probability they are caused by the way the various data have been acquired. Rather, they may be true signals of the periodic activity of Proxima Cen. The MEarth DR7 data folded in phase with the 91.0-day period are shown in the bottom panel of Fig. 9. A sinusoid curve with an amplitude of $8.7 \mathrm{mmag}$ is plotted on top of the observations.

Proxima Cen has also been part of the All-Sky Automated Survey for Supernovae (ASAS-SN) long-term photometric monitoring (Shappee et al. 2014). The ASAS-SN V-band light curve is shown in the bottom panel of Fig. 7. It overlaps with the MEarth DR7 data and extends over an additional period of several months of observations. Both the MEarth DR7 and ASAS-SN light curves cover a total of $3.9 \mathrm{yr}$ of continuous photometric monitoring. There are two obvious features highlighted by the comparison of both light curves: one is that the amplitude of the variations of the ASAS-SN data is larger than that of the MEarth DR7 photometry. This is very likely explained by the fact that the variability of Proxima Cen is more notorious at blue wavelengths. The second feature is that the ASAS-SN photometry shows a decreasing trend towards fainter $V$ magnitudes, which contrasts with the flat nature of the MEarth $R G 715$ data. We note that the state of minimum activity labelled with the letter "A" in previous sections corresponds to epochs with the faintest $V$ magnitudes. Whether this trend is real or an artifact of the ASAS-SN data is not clear to us since the two curves were acquired with different filters. The decreasing trend, which is mostly dominated by the most recent epoch of observations, induces a strong signal/power at small frequencies in the Lomb-Scargle periodogram of the ASAS-SN data. To identify potential peaks at longer frequencies, we removed the 


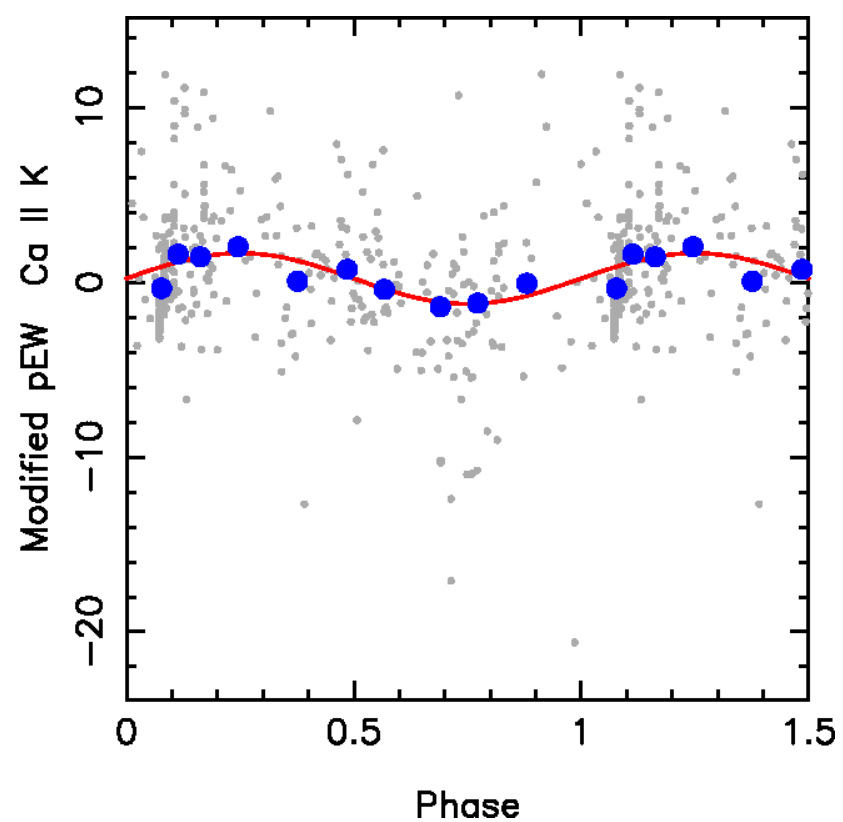

Fig. 6. Median-subtracted pEWs of the CaII K line (grey dots) folded in phase with the period of 90.72 days. The red line depicts the sinusoidal fit to the folded data (amplitude of $1.5 \AA$ ), and the blue dots correspond to the data points averaged with their 21 neighbours. Flares and strongly deviant data points are not included in the diagram.

trend by subtracting a fourth-order polynomial from the original photometry. The resulting Lomb-Scargle periodogram is shown in the bottom panel of Fig. 8, where one strong peak is visible at $96.3 \pm 5.0$ days. After removing this signal from the light curve, there remains only one significant peak at $82.8 \pm 4.0$ days with a FAP above $0.1 \%$.

Within 1- $\sigma$ of the quoted uncertainties, we found the peaks at $\sim 91$ and $\sim 83$ days to be common to all three data sets (HARPS, MEarth, and ASAS-SN), with the peak at $\sim 91$ days being the strongest signal. These two periodicities are likely related to the rotation period of Proxima Cen. The changes in the variability periods could correspond to changes and evolution (appearance and disappearance) of active regions located at different latitudes on the stellar surface. Proxima Cen shows multiple significant periods, which we interpret as differential rotation. On the other hand, we cannot exclude the possibility that the peaks at $\sim 91$ or $\sim 83$ days are another alias of the annual window.

The strong flare, event $\mathrm{B}$, reported in previous sections is reasonably well covered by the MEarth data: photometric observations are available before, during, and after the flare. This is the only flare reported here for which there is such temporal coverage in the MEarth DR7 database. Figure 10 depicts the MEarth photometry corresponding to March 18, 2016; the location of the spectroscopic flare is indicated. The flare event induced a photometric increase of $\geq 45$ mmag in the brightness of Proxima Cen at red optical wavelengths, which is about nine times greater than the amplitude of the rotation curve.

\section{Discussion}

In this paper we analyse the flare activity of Proxima Cen using extended sets of HARPS spectra. We inspect the intensity of the $\mathrm{H}_{\alpha}$ line to study the "flare activity". Pavlenko et al. (2017) showed that the molecular background of the emission lines in the spectrum of Proxima Cen shows rather marginal response to the variations of the activity level. Using this discovery, we
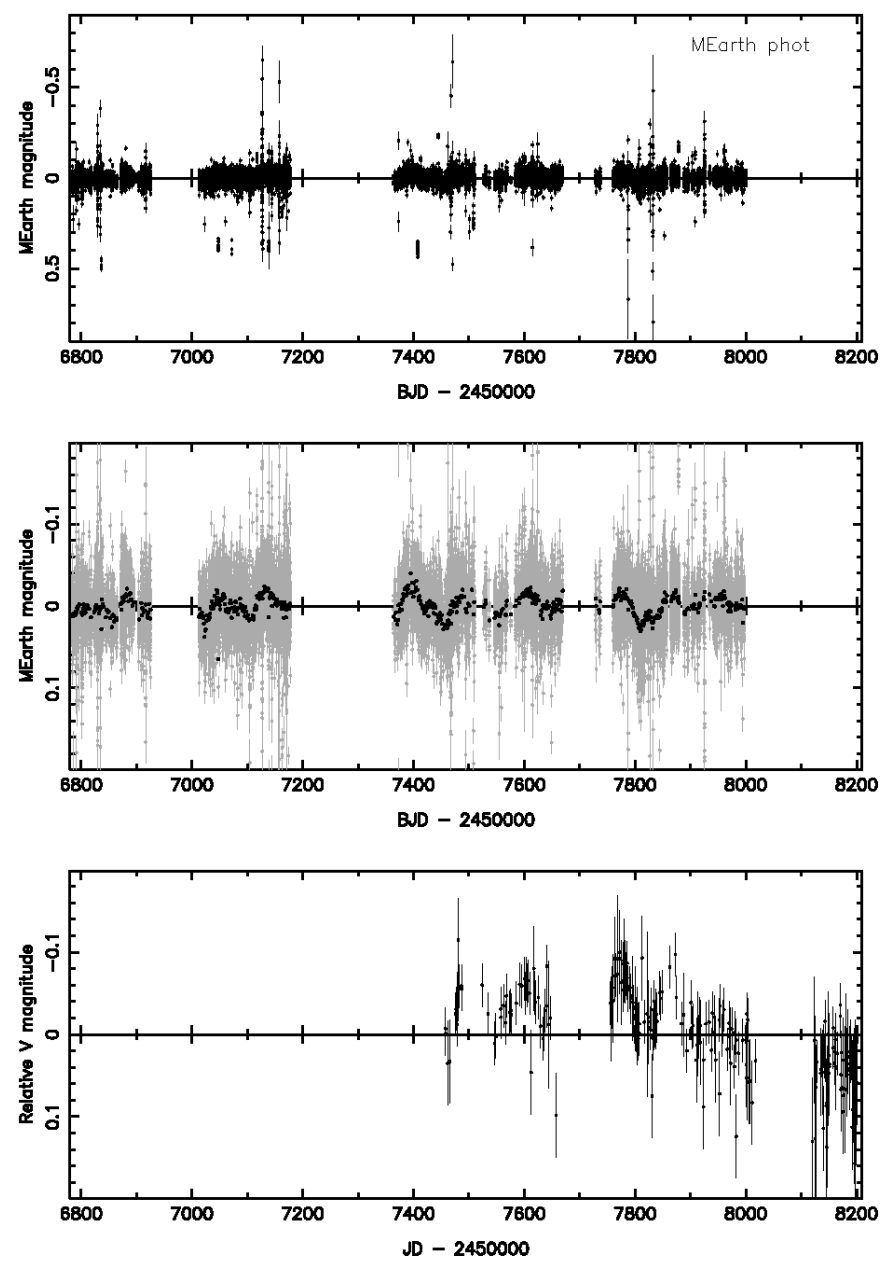

Fig. 7. Top: Proxima Cen photometric light curve (filter $R G 715$ ) delivered by MEarth DR7 (black dots). The data span a period of $3.2 \mathrm{yr}$. Negative magnitude simply brighter states of the star. Middle: enlargement of the MEarth DR7 light curve. Original data are plotted as grey dots. The day-averaged photometry is shown with black dots. Bottom: ASAS-SN light curve (black dots) spanning a time period of $2 \mathrm{yr}$. The mean magnitude of 11.227 mag has been subtracted from the original data for a proper comparison with the MEarth DR7 light curve.

computed the pEW of multiple emission lines and present a detailed analysis of the profiles of several emission lines of interest to study dynamic processes occurring during different flare phases. We find some similarities and differences in the temporal changes of $\mathrm{H}_{\alpha}$ and other emission lines.

The hydrogen and helium emission lines are of special interest. Indeed, these lines form in the flare region, in layers of high temperature $(T>10000 \mathrm{~K})$ in the inner and/or outer chromosphere, where we observe a large dispersion of velocities in the largest flares. HeI lines form in the regions of higher temperatures and their intensities are rather marginal during the minimum activity of Proxima Cen, contrary to $\mathrm{H}_{\alpha}$.

The minima in $\mathrm{pEW}$ of $\mathrm{H}_{\alpha}$ ("A") are just barely fainter than the mean $\mathrm{pEW}$, yet the minima in $\mathrm{H}_{\epsilon}$ are about ten times lower than the mean $\mathrm{pEW}$. This raises the possibility that the blue end of the spectra is prone to very low $\mathrm{S} / \mathrm{N}$ on certain dates, not that the blue emission lines are actually lower on the star.

The $\mathrm{HI}$ emission line $\mathrm{H}_{\epsilon}$ disappears almost completely during the minimum activity of Proxima Cen, an effect that cannot be explained by the low $\mathrm{S} / \mathrm{N}$ on those dates (Sect. 4.6). Indeed, our analysis shows that the $\mathrm{S} / \mathrm{N}$ in the blue part of the spectra containing $\mathrm{CaII} \mathrm{H}$ and $\mathrm{K}$ and $\mathrm{H}_{\epsilon}$ is higher in quiet mode. On the 

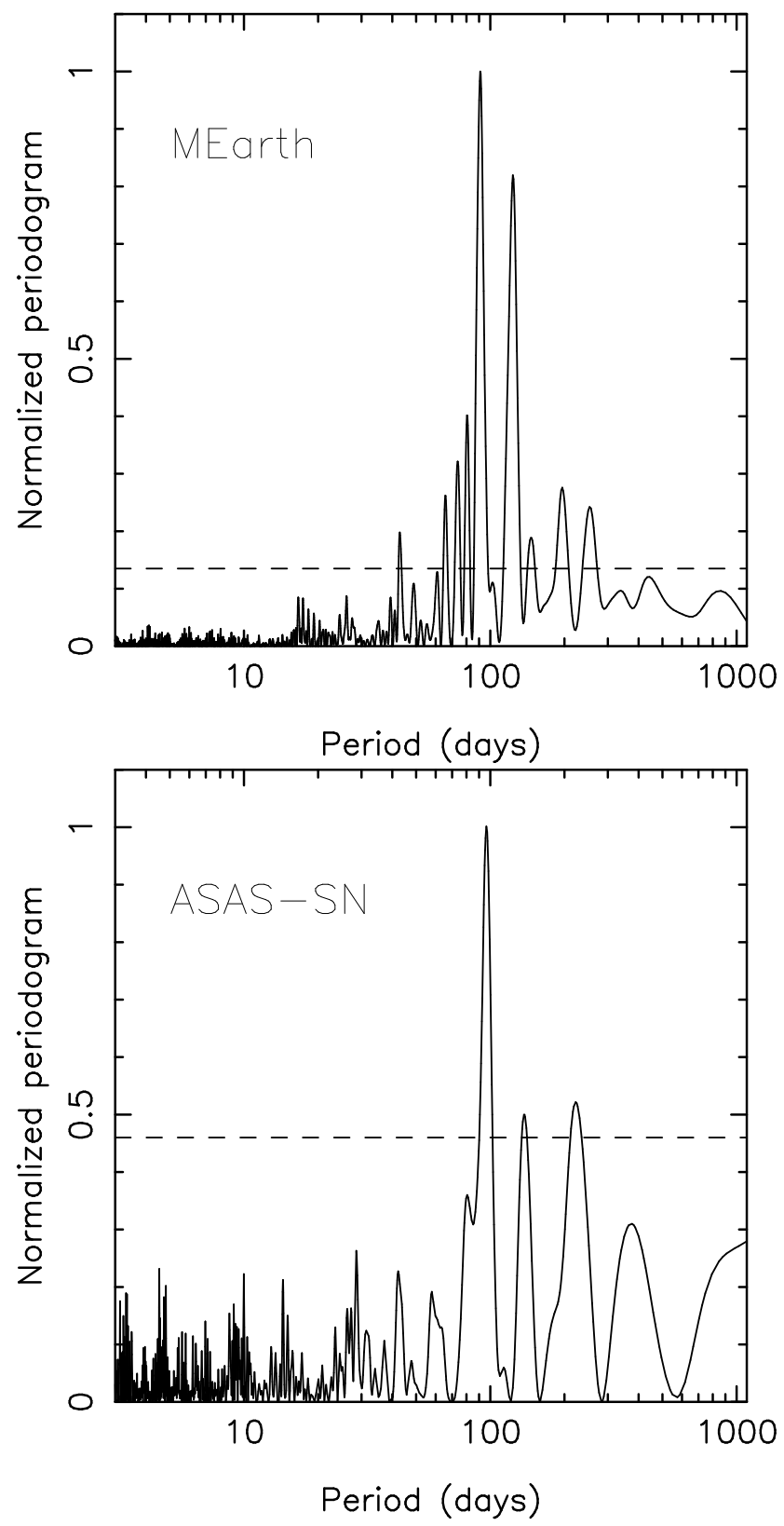

Fig. 8. Top: Lomb-Scargle periodogram of the MEarth DR7 light curve for Proxima Cen. Bottom: Lomb-Scargle periodogram of the ASAS-SN light curve for Proxima Cen after subtraction of the decreasing flux trend. The $0.1 \%$ FAP is indicated by the horizontal dashed lines.

contrary, we see a drop in $\mathrm{S} / \mathrm{N}$ during phases with strong flares. However, at these dates $\mathrm{H}_{\epsilon}$ increases its intensity by several orders of magnitude. Even if the increase of $\mathrm{H}_{\epsilon}$ originates from the drop of the background flux formed in the stellar atmospheres the formally computed $\mathrm{pEW}$ should increase during flares.

We suggest that differences in the physical conditions responsible for the formation of the emission lines in quiet and flare modes may provide a more realistic explanation of these phenomena. Indeed, the Balmer decrement should change in flare mode due to variable physical conditions in the regions where hydrogen forms. A more detailed consideration of this problem is beyond the scope of this paper, but this last possibility cannot be discarded.

Moreover, the formation of the strong emission in the core of the NaI line appears very unusual, as already noted by Pavlenko et al. (2017). Special conditions are needed to create such strong
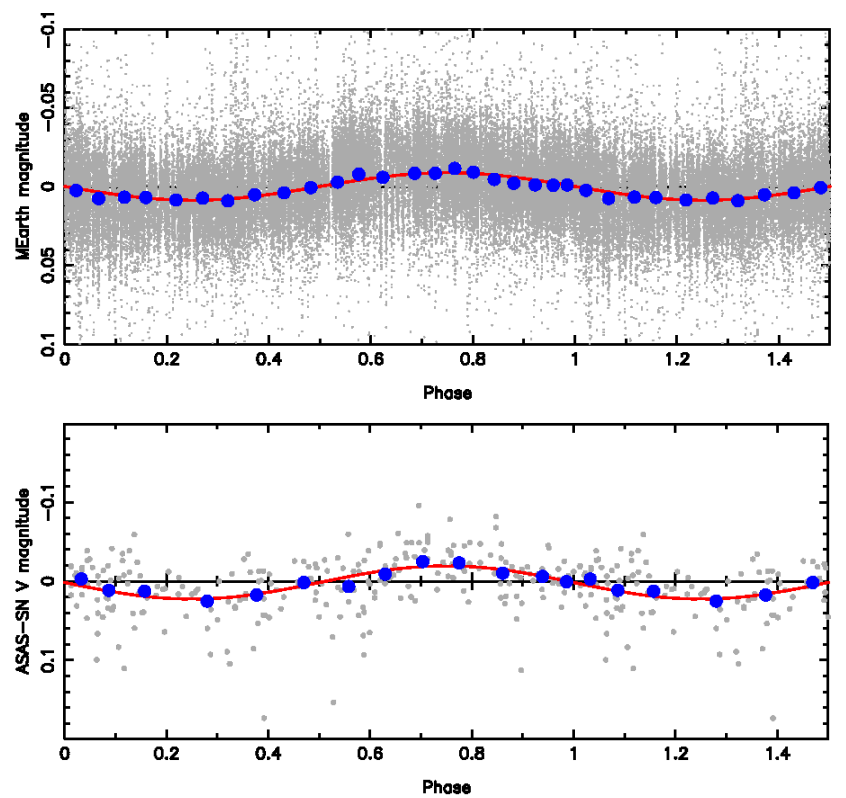

Fig. 9. MEarth DR7 (top) and ASAS-SN (bottom) light curves (grey dots) folded with a period of 91.0 and $96.5 \mathrm{~d}$, respectively. The sinusoid fit to the data is shown with the red curve (amplitudes of 8.7 and $21.0 \mathrm{mmag}$ ). Each blue dot corresponds to the average of 3000 MEarth and 19 ASAS-SN individual data points. We note that the vertical scale is different by a factor of two different between the two panels.

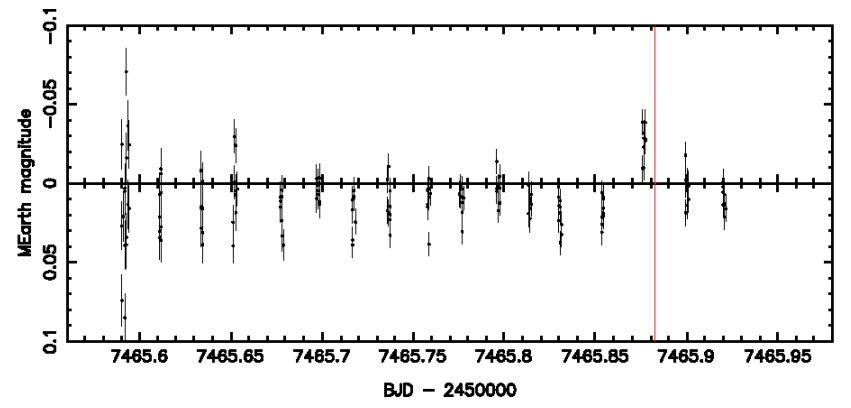

Fig. 10. MEarth DR7 light curve of March 18, 2016. The red vertical line indicates the location of the spectroscopic flare B.

$\mathrm{NaI}$ emission. We see that the half-width of the NaI line shows rather marginal changes during flares. At the same time, the intensity of the core of this line reduces in the strong flare modes while some emission appears in the wings.

Our study is based on the analysis of the pEW and emission line profiles of HI, HeI, CaII, and NaI. Firstly, we looked at their correlation with the strength of $\mathrm{H}_{\alpha}$. We selected $\mathrm{H}_{\alpha}$ as the reference line because it forms in high-temperature (flare) regions. All lines show a good correlation with the strength of $\mathrm{H}_{\alpha}$. The $\mathrm{He}_{4026}$ line shows the weakest correlation with $\mathrm{H}_{\alpha}$, indicating that the processes and regions of the formation of this line differ from other lines. Interestingly, we note that the other HeI line $\mathrm{He}_{5876}$ better follows $\mathrm{H}_{\alpha}$.

From our dataset we selected three dates of minimum and maximum activity of Proxima Cen. During the minimum activity, the spectrum of Proxima Cen does not exhibit any $\mathrm{He}_{5876}$ or $\mathrm{He}_{4026}$ lines. Moreover, the intensity of $\mathrm{H}_{\alpha}$ is reduced by a factor of eight with respect to its maximum values, while the intensity of the CaII lines are 150 times lower. We observe an almost complete disappearance of the lines with the highest excitation during the days of absolute minimum of $\mathrm{H}_{\alpha}$ on $\mathrm{BJD}=8021.4600,8023.4639$, and 8027.4785 (Sect. 4.4). 


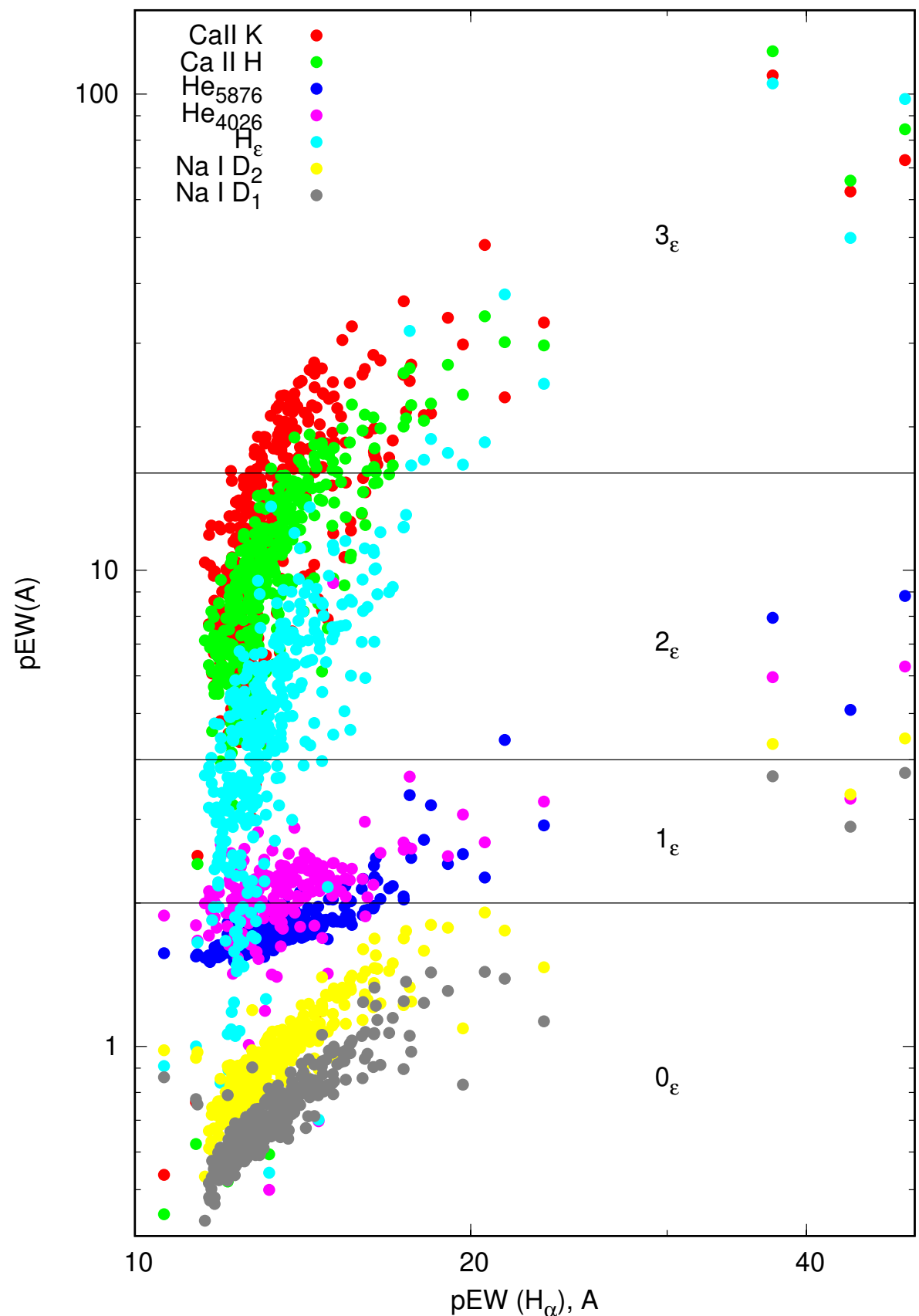

Fig. 11. Correlation of the variations in the $\mathrm{pEW}$ of some emission lines with the $\mathrm{pEW}$ of $\mathrm{H}_{\alpha}$ for all dates. The horizontal lines split the figure with respect to our criteria of the strengths of $\mathrm{H}_{\epsilon}$ (Sect. 1).
Furthermore, the CaII lines almost disappear, as if the star had lost its chromosphere for a while. This period of minimum activity of the chromosphere may last a few days or possibly weeks. Even the strongest of all lines, $\mathrm{H}_{\epsilon}$, is not seen, although this line is entirely formed in the flare regions, contrary to $\mathrm{H}_{\alpha}$ which is still present in the quiet state. The evaporated chromospheric matter in flares becomes cooler during minimum activity, suggesting that the condensed matter absorbs the blue parts of the $\mathrm{NaI}$ emission lines. The emission in $\mathrm{NaI} \mathrm{D}_{1}$ and $\mathrm{D}_{2}$ lines is still present, implying that they likely form in the remnants of the formerly strong chromosphere.

During the periods of strongest activity of Proxima Cen, an extended red wing is present in the $\mathrm{H}_{\alpha}$ line, most likely as a result of the hot matter penetrating into the cooler layers. Strong flares affect the formation region of CaII lines and show the anti-correlation with $\mathrm{H}_{\alpha}$ (Pavlenko et al. 2017). The intensity of the NaI, $\mathrm{D}_{1}$, and $\mathrm{D}_{2}$ lines is reduced during strong flares, but not as much as the CaII lines. Interestingly, different flares yield emission lines of different widths, suggesting that stronger flares occur in the regions of larger turbulent velocity dispersion. Even moderately strong $1_{\epsilon}$ flares produce the notable $\mathrm{He}_{4026}$ and $\mathrm{He}_{5876}$ lines (Fig. 11). We also emphasise that absorption components are seen in the cores of all lines during maximum activity.

We analysed extended sets of spectra obtained on specific dates where flares of different strengths took place. The study of temporal sequences of $\mathrm{H}_{\alpha}$ reveals variability on timescales of $10 \mathrm{~min}$. We observe a few flares at good temporal resolution even though flares may also occur on shorter timescales. All flares show similar structures with several phases, including a slow initialization, a fast phase, a phase of fading, a secondary increase of intensity, followed by another fading phase. Unfortunately, our interpretation of the different phases lacks critical information: firstly, we do not know the precise geometry and size of flare regions. Secondly, we do not spatially resolve the stellar disc, implying that the spectrum of Proxima Cen includes the integrated flux of the whole stellar surface. Finally, flares 
may appear at different parts of the stellar disc and even at the limb, or partially beyond the limb. All these cases of potential variability may produce different shapes of the observed lines.

Fortunately, we have at least four full nights with good time coverage where we see different profiles due to activity. On $\mathrm{BJD}=6417$ we have a flare with a strength of $3_{\epsilon}$ with welldefined temporal behaviour. A similar flare structure may be present on the following day $(\mathrm{BJD}=6418)$, however, the temporal changes here are smoother in time. On the other hand, we see a flare on $\mathrm{BJD}=6418$, at least seen in the $\mathrm{H}_{\epsilon}$ behaviour. These results agree with Davenport et al. (2014) who found that the majority of flares occurring in the atmosphere of the active M4 dwarf GJ 1243 last longer than approximately $30 \mathrm{~min}$ and show multiple peaks depicting the complex nature of flares.

Nevertheless, we see different behaviour of emission lines formed in the flare regions and the chromosphere. Our analyses suggest the presence of a flare region located over the chromosphere, with both regions being bound by a transition zone. Strong flares penetrate the chromospheric layers and partially destroy them reducing the strength of chromospheric lines. In other words, the structure of the flare region is more complicated than in the case of the Sun. Strong emission in the NaI, $D_{1}$, and $\mathrm{D}_{2}$ lines is present during phases of minimum activity, however this emission is affected by absorption of cooling plasma located in formerly hot regions. We believe that these lines form in the lower chromosphere, as their widths show little variation with the level of activity.

We encourage observers to acquire more continuous HARPS observations over several days/weeks to assess the structure, geometry, and evolution of the flare regions. This strategy should also be extended to other bright $\mathrm{M}$ dwarfs in order to compare these patterns in a broader range of sources.

Acknowledgements. The authors kindly thank M.A. Bautista, S.N. Nahar, M.J. Seaton, and D.A. Verner who supplied data compiled in the NIST database. This research has made use of the Simbad and Vizier databases, operated at the Centre de Données Astronomiques de Strasbourg (CDS), and of NASA's Astrophysics Data System Bibliographic Services (ADS). Based on observations collected at the European Organisation for Astronomical Research in the Southern Hemisphere under ESO programme(s) 087.D-0300(A). This research has made use of the services of the ESO Science Archive Facility. This paper makes use of data from the MEarth Project, which is a collaboration between Harvard University and the Smithsonian Astrophysical Observatory. The MEarth Project acknowledges funding from the David and Lucile Packard Fellowship for Science and Engineering and the National Science Foundation under grants AST-0807690, AST-1109468, AST-1616624 and AST-1004488 (Alan T. Waterman Award), and a grant from the John Templeton Foundation. Y.P. thanks financial support from the Fundación Jesús Serra for a 2 month stay (Sept-Oct 2016) as a visiting professor at the Instituto de Astrofísica de Canarias (IAC) in Tenerife. N.L. and V.J.S.B are supported by the AYA2015-69350-C3-2-P program from Spanish Ministry of Economy and Competitiveness (MINECO). J.I.G.H. acknowledges financial support from the Spanish MINECO under the 2013 Ramón y Cajal program MINECO RYC-2013-14875, and A.S.M., J.I.G.H., and R.R.L. also acknowledge financial support from MINECO (program AYA2014-56359-P). We thank the anonymous referee for a thorough review and we highly appreciate the comments and suggestions, which significantly contributed to improving the quality of the publication. Y.P. thanks Language Editor Joshua Neve for the work on the article language.

\section{References}

Abbett, W. P., \& Hawley, S. L. 1999, ApJ, 521, 906

Anglada-Escudé, G., Amado, P. J., Barnes, J., et al. 2016, Nature, 536, 437

Astudillo-Defru, N., Bonfils, X., Delfosse, X., et al. 2015, A\&A, 575, A119

Barnes, S. A. 2003, ApJ, 586, L145

Barnes, S. A., \& Kim, Y.-C. 2010, ApJ, 721, 675

Barnes, J. R., Jeffers, S. V., \& Jones, H. R. A. 2011, MNRAS, 412, 1599
Benedict, G. F., McArthur, B., Nelan, E., et al. 1998, AJ, 116, 429

Berta, Z. K., Irwin, J., Charbonneau, D., Burke, C. J., \& Falco, E. E. 2012, AJ, 144,145

Bonfils, X., Gillon, M., Forveille, T., et al. 2011, A\&A, 528, A111

Bopp, B. W., Grupsmith, G., McMillan, R. S., Vanden Bout, P. A., \& Wootten, H. A. 1973, ApJ, 186, L123

Browning, M. K., Basri, G., Marcy, G. W., West, A. A., \& Zhang, J. 2010, AJ, 139,504

Charbonneau, D., Berta, Z. K., Irwin, J., et al. 2009, Nature, 462, 891

Christian, D. J., Mathioudakis, M., Bloomfield, D. S., Dupuis, J., \& Keenan, F. P. 2004, ApJ, 612, 1140

Collins, J. M., Jones, H. R. A., \& Barnes, J. R. 2017, A\&A, 602, A48

Davenport, J. R. A., Hawley, S. L., Hebb, L., et al. 2014, ApJ, 797, 122

Davenport, J. R. A., Kipping, D. M., Sasselov, D., Matthews, J. M., \& Cameron, C. 2016, ApJ, 829, L31

Delfosse, X., Forveille, T., Perrier, C., \& Mayor, M. 1998, A\&A, 331, 581

Delorme, P., Collier Cameron, A., Hebb, L., et al. 2011, MNRAS, 413, 2218

Doyle, L., Ramsay, G., Doyle, J. G., Wu, K., \& Scullion, E. 2018, MNRAS, 480, 2153

Fletcher, L., Dennis, B. R., Hudson, H. S., et al. 2011, Space Sci. Rev., 159, 19

Fuhrmeister, B., Lalitha, S., Poppenhaeger, K., et al. 2011, A\&A, 534, A133

Gershberg, R. E. 2005, Solar-Type Activity in Main-Sequence Stars (Berlin: Springer)

Gershberg, R. E., \& Shakhovskaia, N. I. 1983, Ap\&SS, 95, 235

Gomes da Silva, J., Santos, N. C., Bonfils, X., et al. 2012, A\&A, 541, A9

Hawley, S. L., \& Pettersen, B. R. 1991, ApJ, 378, 725

Hawley, S. L., Gizis, J. E., \& Reid, I. N. 1996, AJ, 112, 2799

Hawley, S. L., Davenport, J. R. A., Kowalski, A. F., et al. 2014, ApJ, 797, 121

Heise, J., Brinkman, A. C., Schrijver, J., et al. 1975, ApJ, 202, L73

Howard, W. S., Tilley, M. A., Corbett, H., et al. 2018, ApJ, 860, L30

Irwin, J., Berta, Z. K., Burke, C. J., et al. 2011, ApJ, 727, L56

Jenkins, J. S., Jones, H. R. A., Tuomi, M., et al. 2017, MNRAS, 466, 443

Kiraga, M., \& Stepien, K. 2007, Acta Astron., 57, 149

Kowalski, A. F., Hawley, S. L., Hilton, E. J., et al. 2009, AJ, 138, 633

Léger, A., Rouan, D., Schneider, J., et al. 2009, A\&A, 506, 287

Lomb, N. R. 1976, Ap\&SS, 39, 447

Mestel, L. 1984, in Cool Stars, Stellar Systems, and the Sun, eds. S. L. Baliunas

\& L. Hartmann, Lect. Notes Phys. (Berlin: Springer Verlag), 193, 49

Mestel, L., \& Spruit, H. C. 1987, MNRAS, 226, 57

Morin, J., Donati, J.-F., Forveille, T., et al. 2008, MNRAS, 384, 77

Morin, J., Donati, J.-F., Petit, P., et al. 2010, MNRAS, 407, 2269

Newton, E. R., Irwin, J., Charbonneau, D., et al. 2016, ApJ, 821, 93

Newton, E. R., Irwin, J., Charbonneau, D., et al. 2017, AAS Meeting Abstracts, 229, 131.03

Parker, E. N. 1955, ApJ, 122, 293

Paulson, D. B., Allred, J. C., Anderson, R. B., et al. 2006, PASP, 118, 227

Pavlenko, Y., Suárez Mascareño, A., Rebolo, R., et al. 2017, A\&A, 606, A49

Pojmanski, G. 1997, Acta Astron., 47, 467

Pojmanski, G., \& Maciejewski, G. 2004, Acta Astron., 54, 153

Queloz, D., Bouchy, F., Moutou, C., et al. 2009, A\&A, 506, 303

Quintana, E. V., \& Barclay, T. 2014, AAS Meeting Abstracts, 224, 113.06

Reiners, A., \& Basri, G. 2008, ApJ, 684, 1390

Rivera, J. L., Loinard, L., Dzib, S. A., et al. 2015, ApJ, 807, 119

Scargle, J. D. 1982, ApJ, 263, 835

Schmidt, S. J., Prieto, J. L., Stanek, K. Z., et al. 2014, ApJ, 781, L24

Shappee, B. J., Prieto, J. L., Grupe, D., et al. 2014, ApJ, 788, 48

Skumanich, A. 1972, ApJ, 171, 565

Suárez Mascareño, A., Rebolo, R., González Hernández, J. I., \& Esposito, M. 2015, MNRAS, 452, 2745

Suárez Mascareño, A., Rebolo, R., González Hernández, J. I., \& Esposito, M. 2016, MNRAS, 457, 2604

Suárez Mascareño, A., Rebolo, R., González Hernández, J. I., \& Esposito, M. 2017, MNRAS, 468, 4772

Thomas, R. N. 1957, ApJ, 125, 260

Thompson, A. P. G., Watson, C. A., de Mooij, E. J. W., \& Jess, D. B. 2017, MNRAS, 468, L16

Torres, G., Kipping, D. M., Fressin, F., et al. 2015, ApJ, 800, 99

Udry, S., Bonfils, X., Delfosse, X., et al. 2007, A\&A, 469, L43

Walkowicz, L. M., Basri, G., Batalha, N., et al. 2011, AJ, 141, 50

Wargelin, B. J., Saar, S. H., Pojmański, G., Drake, J. J., \& Kashyap, V. L. 2017, MNRAS, 464, 3281

West, A. A., Weisenburger, K. L., Irwin, J., et al. 2015, ApJ, 812, 3

Wright, D. J., Wittenmyer, R. A., Tinney, C. G., Bentley, J. S., \& Zhao, J. 2016, ApJ, 817, L20

Yokoyama, T., \& Shibata, K. 1998, ApJ, 494, L113

York, D. G., Adelman, J., Anderson, Jr. J. E., et al. 2000, AJ, 120, 1579

Zhang, Q. M., Li, D., \& Ning, Z. J. 2016, ApJ, 832, 65 